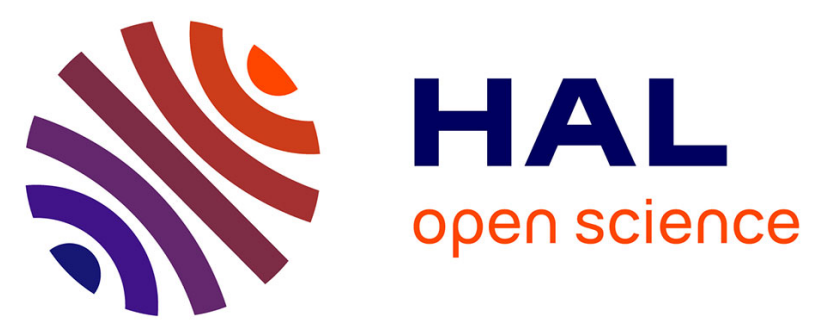

\title{
Vibro-impact dynamics of a two-degree-of freedom periodically-forced system with a clearance: Diversity and parameter matching of periodic-impact motions
} G.W. Luo, X.H. Lv, Y.Q. Shi

\section{- To cite this version:}

G.W. Luo, X.H. Lv, Y.Q. Shi. Vibro-impact dynamics of a two-degree-of freedom periodically-forced system with a clearance: Diversity and parameter matching of periodic-impact motions. International Journal of Non-Linear Mechanics, 2014, 65, pp.173 - 195. 10.1016/j.ijnonlinmec.2014.04.013 . hal01403576

\author{
HAL Id: hal-01403576 \\ https://hal.science/hal-01403576
}

Submitted on 26 Nov 2016

HAL is a multi-disciplinary open access archive for the deposit and dissemination of scientific research documents, whether they are published or not. The documents may come from teaching and research institutions in France or abroad, or from public or private research centers.
L'archive ouverte pluridisciplinaire HAL, est destinée au dépôt et à la diffusion de documents scientifiques de niveau recherche, publiés ou non, émanant des établissements d'enseignement et de recherche français ou étrangers, des laboratoires publics ou privés. 


\title{
Vibro-impact dynamics of a two-degree-of freedom periodically-forced system with a clearance: Diversity and parameter matching of periodic-impact motions
}

\author{
G.W. Luo, X.H. Lv, Y.Q. Shi \\ Key Laboratory of System Dynamics and Reliability of Rail Transport Equipment of Gansu Province, Lanzhou Jiaotong University, Lanzhou 730070, PR China
}

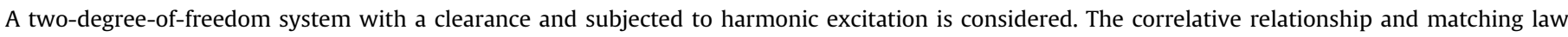

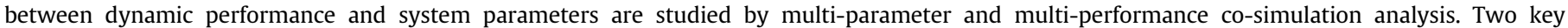

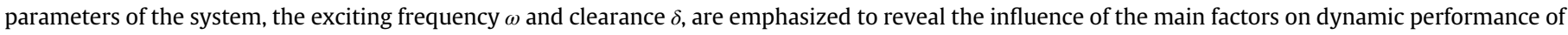

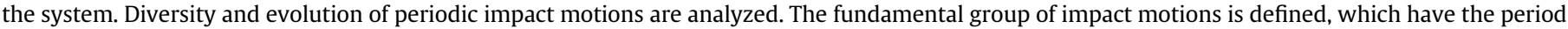

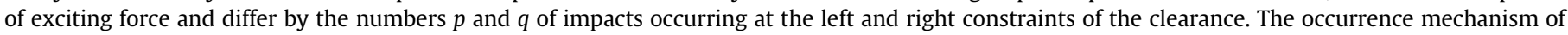

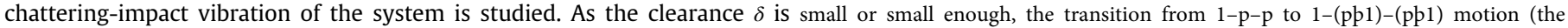

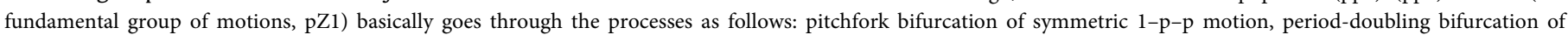

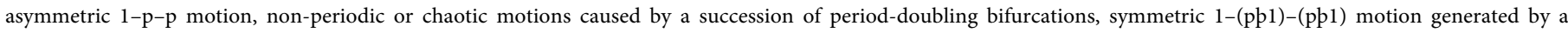

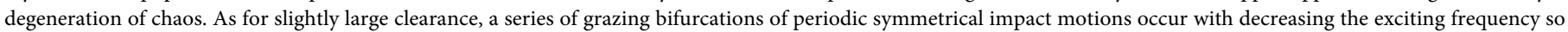

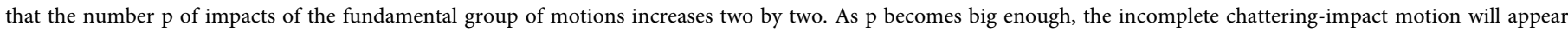

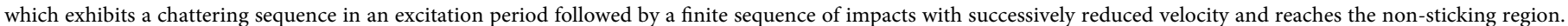

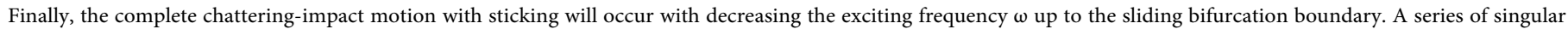

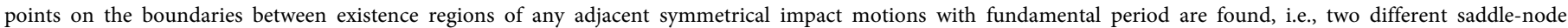

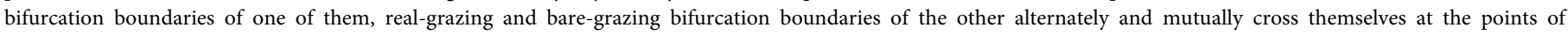

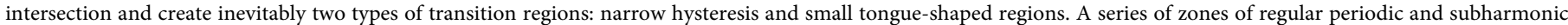

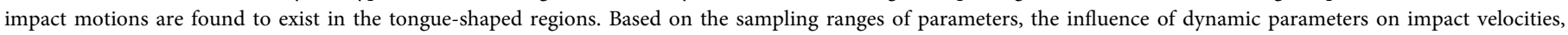
existence regions and correlative distribution of different types of periodic-impact motions of the system is emphatically analyzed.
\end{abstract}

\section{Introduction}

The clearances or constraints among mechanical components exist inevitably in mechanical systems due to the restriction on technological level in the mechanical machining process, the demand for mechanical design and assembly errors, the need for the heat-expansion and cold-contraction of some part of mechanical components or other reasons. The existence of the clearances or motion limiting constraints is bound to bring about the vibroimpact effects which have a great influence on performance of mechanical equipments, increase noise levels and intensify fatigue and abrasion of mechanical parts. The non-smooth natures of phase trajectories caused by the impacts complicate the dynamic analysis of such systems inevitably, but they can be described theoretically and numerically by the discontinuities in good agreement with reality. It is necessary to be able accurately to model the dynamics of the mechanical systems with clearances or motion limiting constraints from the multi-parameter level, so as to utilize effectively and enlarge profitable effects by reasonable 
parameter matching design, to furthest minimize the influence of unfavorable factors such as abrasion, chattering-impact, high noise levels, etc. The broad interest in revealing dynamical performance of vibro-impact systems is reflected by an ever increasing amount of investigations devoted to this aspect. Several effective methods of qualitative, numerical and experimental analyses have been proposed and their validity has been demonstrated by different vibro-impact models in the past several years. Dynamics of different types of vibratory systems with clearances or motion limiting constraints, including detailed observations about stability and bifurcations using numerical simulations and qualitative analyses, were partly reported in Refs. [1-10]. Shaw and Holmes [1] studied a single-degree-of-freedom vibro-impact system by casting the traditional approaches used for analyzing the periodic responses in the system in the form of a more general Poincaré map. The notable work showed how even a very simple impact oscillator could display the full range of typical nonlinear behavior: saddle node and flip bifurcations, multiple coexisting attracting solutions, chaos, etc. Periodic and chaotic behavior of a threshold-limited two-degree-of-freedom system was analyzed by Aidanpää and Gupta [2]. Souza and Caldas [3] put forward an algorithm of the spectrum of Lyapunov exponents of attractors of a vibro-impact system for interpreting bifurcations and chaotic transitions. Peterka and Kotera [4] studied the appearance and characteristics of intermittency chaos of an impact oscillator occurring near saddle-node bifurcation boundaries of neighboring fundamental motions with two different impacts in an excitation period. Pavlovskaia and Wiercigroch $[5,6]$ developed an efficient semi-analytical method to study the percussive drilling principle for the impact systems with drift and accurately predicted a range of control parameters for which the best progression rates were obtained. Quinn and Bairavarasu [7] considered a three-mass collision model with compliant contacts, a two-dimensional map is derived to describe initial conditions that lead to pairwise sequences, and the variation in the final velocities is characterized in terms of the initial configuration of the system. Luo [8] determined analytically and numerically stability and bifurcation conditions for the LR model motion in an impact oscillator, for which the regions are developed in parameter space. Leine [9] presented an asymptotic approximation method for the critical restitution coefficient of a parametrically excited impact oscillator and described its dynamics by an unilaterally constrained Hill's equation. More studies on vibro-impact dynamics were summarized in Ibrahim's monograph [10]. A special feature of vibroimpact systems that might prove to be useful in the current study is the instability caused by low-velocity collisions, which is the so-called grazing effect. The pioneer work in this field was done by Nordmark [11], who developed systematic methods for investigating grazing dynamics and their attendant bifurcations, providing the results which laid the foundation for many subsequent studies. Thereafter, this work has been further expanded by discontinuitygeometry approaches of a series of impact maps, where the increasingly thorough investigations of grazing bifurcations of such maps have been reported in Refs. [12-25]. The chatteringimpact vibration denotes an accumulation of impact events in finite time (or a motion period), and sticking is where the dynamics becomes finally constrained to the impact surface as the impact attenuation ends. Detailed studies of chattering-impact vibration with sticking were carried out for the vibro-impact systems in Ref. [26-35]. Rising events occurring in multi-sticking solutions are discussed by Wagg [29,30], the study on simulation and stability analysis of impacting systems with complete chattering has been further expanded by Nordmark and Piiroinen [31]. de Souza and Caldas [32] numerically investigated the basins of attraction of coexisting periodic and chaotic attractors in a gear-rattling impact model. Luo [33,34] presented an idealized, piecewise linear system to model non-smooth vibration of gear transmission arising from impacts between the gear teeth and predicted analytically occurrence of the periodic impact-sticking motions. Hősa and Champneysb [35] considered a mechanical model of a pressure relief valve and analyzed the geometry of the chattering region via the computation of several pre-images of the grazing set. The vibro-impact systems display many of the complex dynamical features near critical points of high codimension bifurcations; in particular, different types of codimension two bifurcations and parameter unfolding associated with the interaction of saddle-node and grazing bifurcations [23], double flip bifurcation [36], Hopf-flip bifurcation [37], Hopf-pitchfork bifurcation and double Neimark-Sacker bifurcation [38], codimensiontwo grazing bifurcations [39,40], grazing-sliding bifurcation [41], etc., were studied by numerical simulations and qualitative analyses in recent years. Several methods of experimental analyses have been developed for some impact models in Refs. [42-46]. Wiercigroch and Sin [42] performed an experimental study of base excited symmetrically piecewise linear oscillator for observing and proving symmetric and asymmetric periodic-impact motions and their attendant bifurcations. Jin and $\mathrm{Hu}$ [43] designed an experimental setup consisting of both vibro-impacting cantilever beams with lumped mass blocks for testing various types of vibro-impact motions such as subharmonic, superharmonic, quasi-periodic and chaotic motions, etc. Quinn [44] considered the effect of external forces during finite duration collisions using an incremental model of impact. Based on the results of the experimental system and the incremental model, the standard algebraic model of restitution is modified to include these finite duration effects. Nguyen et al. [45] designed an experimental scheme of vibro-impact rig to mimic a mole penetration through the soil, which was based on electromechanical interactions of a conductor with an oscillating magnetic field. Sitnikova and Pavlovskaia [46] studied bifurcations and chaotic attractors of an impact oscillator with a shape memory alloy constraint. The evolution of chaotic attractors is recorded experimentally, and changes in the structure of the attractors are shown. Along with the requirement of engineering application and the increasing of basic research on non-smooth dynamics, vibro-impact dynamics has been applied to a wide range of practical mechanical systems for finding the correlative relationship between dynamic performance and model parameters, e.g., wheel-rail impacts of railway coaches [47,48], vibrating hammer [49], pile driver [50,51], ground moling dynamics [52], mill rolls [53], the fly-wheel model of the bouncing ball [54], link mechanism [55], ultrasonic percussive drilling [56,57], the vibro-impact capsule system [58], Jeffcott rotors with bearing clearance [59-61], impact dampers [62,63], excited pendula with impacts [64], high frequency vibro-impact drilling [65], pipes conveying [66], vibro-impact interaction of ships with ice [67], gears transmission systems [32-34,68-71], etc. The dynamical models designed in Refs. [47-71] have proved to be useful for revealing dynamical performance of such practical systems and the related studies have provided much information of the fundamental nature that broadened the scope of knowledge on the nonsmooth motions of mechanical systems with impacts.

It is important to note, that most studies of vibro-impact dynamics are based on single-parameter bifurcation analysis in the past several years. Very few have considered multi-performance, multi-process coupling and multi-parameter simulation analysis and collaborative optimization for research on vibroimpact dynamics. The purpose of the present study is to focus attention on multi-performance and multi-parameter simulation analysis for dynamics of a two-degree-of-freedom periodicallyforced system with a clearance represented by two symmetric rigid stops. The correlative relationship between dynamic performance and system parameters is particularly studied to find their 
reasonable matching law. Two key parameters of the system, the exciting frequency $\omega$ and clearance $\delta$, are emphasized to analyze the influence of the main factors on dynamic performance of the system in Section 3, and diversity and evolution of periodic-impact motions are discussed. As for small clearance, the transition between adjacent fundament motions basically goes through three processes with decrease in $\omega$, which are epitomized as follows: pitchfork bifurcation of symmetric $1-p-p$ motion; period-doubling bifurcation of asymmetric $1-p-p$ motion; non-periodic or chaotic motions caused by a succession of period-doubling bifurcations; symmetric $1-(p+1)-(p+1)$ motion generated by a degeneration of chaos. As for slightly large clearance, a series of grazing bifurcations occur with decreasing the exciting frequency so that the number $p$ of impacts of the fundamental group of motions increases two by two. As $p$ becomes big enough, the system will exhibit an incomplete chattering sequence in an excitation period followed by a finite sequence of impacts with successively reduced velocity. Finally, complete chattering-impact motion with sticking will occur with decreasing the exciting frequency $\omega$ up to the sliding bifurcation boundary. A series of singular points on the critical lines between adjacent fundamental motions are found, at which saddle-node bifurcation boundaries of one of them and real-grazing and bare-grazing bifurcation boundaries of the other alternately and mutually cross themselves. Consequently, two types of transition regions are created inevitably by the singular points, i.e., hysteresis and small tongue-shaped regions. A series of zones of subharmonic impact motions are found in the tongueshaped regions. Based on the sampling ranges of parameters, the influence of system parameters on impact velocities, existence regions and correlative distribution of different types of periodicimpact motions of the system is emphatically analyzed in Sections 4 and 5.

\section{Mechanical model}

The mechanical model of a two-degree-of-freedom periodically-forced system with a clearance represented by two symmetric rigid stops is shown in Fig. 1. The vibration displacements of the mass blocks with masses $M_{1}$ and $M_{2}$ are expressed by $X_{1}$ and $X_{2}$, respectively. Two mass blocks are connected by a linear spring with stiffness $K_{1}$ and a linear viscous dashpot with damping constant $C_{1}$, and the mass block $M_{2}$ is attached to the supporting base by the linear spring $K_{2}$ and linear viscous dashpot $C_{2}$. The exciting forces acting on the mass blocks are harmonic with amplitudes $P_{1}$ and $P_{2}$. The exciting frequency and phase angle are the same for two mass blocks, which are respectively represented by $\Omega$ and $\tau$. The vibration amplitude of the system is restricted by a clearance. For small forcing amplitudes the system exhibits simple linear oscillation. With increase in the amplitude, the mass block $M_{1}$ eventually begins to hit the rigid stops of the clearance situated in certain distances $B$ and $-B$ from the equilibrium position of the mass block. Consequently, the contacts of the mass block with the stops result in discontinuous changes in the motion properties of the system. The kinetic energy of the system is dissipated partially on the impact. The presence of the

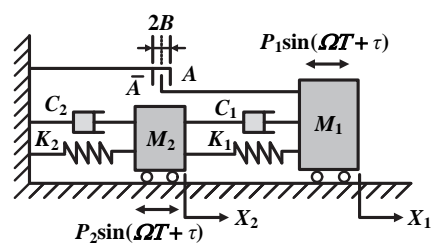

Fig. 1. Schematic of a two-degree-of-freedom periodically-forced system with a clearance. clearance and impact leads to non-smooth nature of motion trajectory of the system and complicates its dynamic analysis. The impact is generally inelastic and modeled by a coefficient of restitution with $0<R<1$. For the simplicity of the induction and summarization, the dynamic analysis can be considered in a dimensionless form - in the following work, we assume all parameters and variables are dimensionless, which are given by

$$
\begin{aligned}
& \mu_{m}=\frac{M_{2}}{M_{2}+M_{1}}, \mu_{k}=\frac{K_{2}}{K_{2}+K_{1}}, \mu_{c}=\frac{C_{2}}{C_{2}+C_{1}}, \\
& \zeta=\frac{C_{1}}{2 \sqrt{K_{1} M_{1}}}, f_{20}=\frac{P_{2}}{P_{2}+P_{1}}, \omega=\Omega \sqrt{\frac{M_{1}}{K_{1}}} \\
& \delta=\frac{B K_{1}}{P_{1}+P_{2}}, t=T \sqrt{\frac{K_{1}}{M_{1}}}, x_{i}=\frac{X_{i} K_{1}}{P_{1}+P_{2}}, i=1,2 .
\end{aligned}
$$

Eq. (1) allows us to make a conclusion that the sampling range of partial dimensionless parameters can be easily identified as $\mu_{m} \in(0,1), \mu_{k} \in(0,1), \mu_{c} \in(0,1), f_{20} \in[0,1]$ and $R \in(0,1)$. It is important to note that the selection of the dimensionless parameters and variables is technically convenient to the induction and summarization of dynamics of the system.

Between any two adjacent impacts, dynamics of the twodegree-of-freedom periodically-forced system is smooth and linear so that its motion process can be governed by the following non-dimensional equations:

$$
\begin{aligned}
& {\left[\begin{array}{cc}
1 & 0 \\
0 & \frac{\mu_{m}}{1-\mu_{m}}
\end{array}\right]\left\{\begin{array}{l}
\ddot{x}_{1} \\
\ddot{x}_{2}
\end{array}\right\}+\left[\begin{array}{cc}
2 \zeta & -2 \zeta \\
-2 \zeta & \frac{2 \zeta}{1-\mu_{c}}
\end{array}\right]\left\{\begin{array}{l}
\dot{x}_{1} \\
\dot{x}_{2}
\end{array}\right\}+\left[\begin{array}{cc}
1 & -1 \\
-1 & \frac{1}{1-\mu_{k}}
\end{array}\right]\left\{\begin{array}{l}
x_{1} \\
x_{2}
\end{array}\right\}} \\
& \quad=\left\{\begin{array}{c}
1-f_{20} \\
f_{20}
\end{array}\right\} \sin (\omega t+\tau), \quad\left(\left|x_{1}\right|<\delta\right),
\end{aligned}
$$

Immediately after the impact occurs, for $\left|x_{1}\right|=\delta$, a discontinuous change in the velocity of the mass block $M_{1}$ occurs and results highly in nonlinear and non-smooth properties, which is determined by the impact law

$\dot{x}_{1 A+}=-R \dot{x}_{1 A-}, \quad\left(x_{1}=\delta\right) ; \quad \dot{x}_{1 \bar{A}+}=-R \dot{x}_{1 \bar{A}-}, \quad\left(x_{1}=-\delta\right)$.

where the subscript signs "-" and "+" denote the states just before and after the impact, respectively; the subscripts $A$ and $\bar{A}$ represent respectively the impacts occurring at the right and left stops of the clearance.

We can conveniently and visually describe different types of periodic-impact motions of the system by the symbol $n-p-q$, where $p$ and $q$ mean the number of impacts occurring at the left $\bar{A}$ and right $A$ constraints respectively, and $n$ represents the number of the exciting force period in the motion period $T_{1}=2 n \pi / \omega$. At small forcing amplitudes a periodic steady state solution of the system will simply be the solution of Eq. (2) with no impacts at all. $p=0$ and $q=0$ symbolize the impactless motion, which exists for enough large clearance $\delta$ (or slightly larger clearances and higher exciting frequencies) and behaves as that of a linear forced vibration system. The quantity $\beta=(p+q) / n$ is of theoretic and practical significance for us to discuss and research thoroughly some dynamic performance on the parameter design of the periodically-forced system with a clearance, which expresses the average impact number during the exciting period.

More generally, a map derived from Eqs. (2) and (3) is used to study the correlative relationship and matching law between dynamics and parameters of the non-smooth system. There is a design technique of the map based on the impact number occurring at a certain stop of the clearance. Consequently, an impact map can be constructed; it is also called impact Poincaré map. This means that each iterate of the map corresponds to the mass block $M_{1}$ impacting the rigid stop once. As the Poincare section $\sigma_{p}=\left\{\left(x_{1}, \dot{x}_{1}, x_{2}, \dot{x}_{2}, t\right) \in \mathbf{R}^{4} \times \mathbf{S}, \quad x_{1}=\delta, \quad \dot{x}_{1}=\dot{x}_{1 A+}\right\}$ related to the motion state of the vibro-impact system just immediately 
after the impact occurring at stop $A$, is chosen, we can construct the Poincare map of the periodically-forced system with a clearance

$X^{(i+1)}=\tilde{f}\left(\nu, X^{(i)}\right)$,

where $X \in \mathbf{R}^{4}, X^{(i)}=\left(\tau^{(i)}, x_{2}^{(i)}, \dot{x}_{1 A+}^{(i)}, \dot{x}_{2}^{(i)}\right)^{\mathrm{T}}, \quad X^{(i+1)}=\left(\tau^{(i+1)}, x_{2}^{(i+1)}\right.$, $\left.\dot{x}_{1 A+}^{(i+1)}, \dot{x}_{2}^{(i+1)}\right)^{\mathrm{T}}, v$ are real parameters, $v \in \mathbf{R}^{m}, m=1$ or $2 \ldots$ or 8.

It is important to note that the Poincare section $\sigma_{n}=$ $\left\{\left(x_{1}, \dot{x}_{1}, x_{2}, \dot{x}_{2}, t\right) \in \mathbf{R}^{4} \times \mathbf{S}, \quad x_{1}=x_{1 \min }, \bmod (t=2 \pi / \omega)\right\}$ may be also considered to construct the other map. Consequently, the impact number $q$ of $n-p-q$ motion can be determined easily by the impact map corresponding to $\sigma_{p}$ and number $n$ of the exciting force periods in the motion period be ascertained by the map related to $\sigma_{n}$. This means that $n-p-q$ motion can be described totally by the two maps. An analytic derivation is made explicitly for the determination of the group of symmetric $n-1-1$ motions and the implicit expression of disturbed map such motions is analytically derived in Ref. [34]. A simplified stability analysis presumes the linear dependence between small differences of the system state from symmetric n-1-1 motion at two succeeding impacts occurring at the same stop. The stability and local bifurcations of the group of symmetric $n-1-1$ motions can be ascertained by computing and analyzing eigenvalues of Jacobian matrix of disturbed map. Correspondingly, saddle-node, perioddoubling, pitchfork and Neimark-Sacker bifurcation boundaries of symmetric n-1-1 motions, in the double-parameter plane, are determined, near which a deeper understanding of the system behavior can be provided.

Here a special case that the impact mass contacts the rigid stop with almost zero before-velocity, i.e., the bare touching, is worth to be emphasized, which causes real or bare-grazing bifurcation of symmetric $1-p-p$ motion and creates two types of transition regions (hysteresis or tongue-shaped regions) in low exciting frequency range.

Let $\bar{F}$ represent a resultant force, which consists of spring restoring force, damping force and sinusoidal excitation acted on the mass block $M_{1}$ and is expressed by

$\bar{F}=2 \zeta\left(\dot{x}_{2}-\dot{x}_{1}\right)+\left(x_{2}-x_{1}\right)+\left(1-f_{20}\right) \sin (\omega t+\tau)$,

If the mass block $M_{1}$ contacts the rigid stop $A$ with almost zero before-velocity and the resultant force $\bar{F}$ is in the positive direction, in an instant immediately after the $i$-th impact contact, i.e.,

$\bar{F}=2 \zeta\left(\dot{x}_{2}^{(i)}\right)+\left(x_{2}^{(i)}-\delta\right)+\left(1-f_{20}\right) \sin \left(\omega t+\tau^{(i-1)}\right) \geq 0$,

the mass $M_{1}$ is stuck to the stop $A$ until the resultant force $\bar{F}$ changes its direction (sign). The change from the motion of mass block $M_{1}$ to its sticking represents a reduction in the degree of freedom of the system from 2 to 1 . Accordingly, the change denotes also a reduction in the dimensions of the map from 4 to 3 . The presence of the sticking phase leads to the segmentation and singularity of the map and complicates the dynamic analysis of the system. If the mass block contacts the rigid stop $A$ with zero velocity and the resultant force $\bar{F}$ is in the negative direction immediately after the $i$-th impact contact, i.e.,

$\bar{F}=2 \zeta\left(\dot{x}_{2}^{(i)}\right)+\left(x_{2}^{(i)}-\delta\right)+\left(1-f_{20}\right) \sin \left(\omega t+\tau^{(i-1)}\right)<0$,

the mass block $M_{1}$ spends no time in sticking to the stop, the force $\bar{F}$ immediately drives the transient-static mass block $M_{1}$ off the stop.

\section{Dynamical characteristics of the periodically-forced system with symmetric rigid stops}

The main aim of this paper is the characterization of dynamics of the non-smooth vibratory system to reveal the correlative relationship and matching law between dynamic performance and system parameters. Based on the definition of dimensionless parameters and variables in Eq. (1), dynamics of the periodicallyforced impact system is governed by eight dimensionless parameters: $\mu_{m}, \mu_{k}, \mu_{c}, \zeta, f_{20}, R, \delta$ and $\omega$. Dynamics of the system mainly depends on the relative clearance and the exciting frequency. Two key parameters of the system, the exciting frequency $\omega$ and clearance $\delta$, are firstly chosen to analyze the influence of the main factors on its dynamic characteristics. It is important to analyze the detailed influence of each parameter on dynamics of the system to obtain parameter sensitivity and reasonable parameter matching. It is of special interest to acquire the diversity and evolution of periodic-impact motions of the system on the basis of the sampling ranges of parameters. The value range of partial dimensionless parameters are easily ascertained by Eq. (1). The sampling ranges of some parameters are identified as $\mu_{m} \in(0,1)$, $\mu_{k} \in(0,1), \mu_{c} \in(0,1), f_{20} \in[0,1]$ and $R \in[0,1]$. Taking the dimensionless parameters: $\mu_{m}=0.5, \mu_{k}=0.5, \mu_{c}=0.5, \zeta=0.1, f_{20}=0$ and $R=0.8$ as the criterion, we analyze the determinants of system parameters and the influence of them on dynamic performance. Existence regions of different types of periodic-impact motions of the system, associated with the criterion parameters, are presented in the $(\omega, \delta)$-parameter plane plotted in Fig. 2(a). Periodicimpact motions ascertained by numerical analyses are characterized and marked by the symbol $n-p-q$ and corresponding colors. The local amplification and detail of Fig. 2 are demonstrated in Fig. 3. Moreover, 1-CIS is used to denote complete chatting-impact periodic motion with sticking (i.e., $1-\bar{p}-\bar{p}$ motion) in Figs. 2 and 3, symmetric and asymmetric 1-1-1 motions are respectively expressed by the symbols $1-1-1_{\mathrm{S}}$ and $1-1-1_{\mathrm{AS}}$, and $\mathrm{PF}, \mathrm{PB}$, sub and sup N-S Bif represent in proper order successively pitchfork, period doubling, supercritical and subcritical Neimark-Sacker bifurcations. As for large $\delta$, symmetric 1-1-1 motion can be found only around the first natural frequency. At other exciting frequencies the system exhibits 1-0-0 impactless motion. With decrease in $\delta$, the window of symmetric 1-1-1 motion becomes wide for large clearance; becomes narrow for slight large clearance; moves towards higher exciting frequencies for small clearance. At small $\delta$, complete chattering-impact and fundamental $1-p-p \quad(p \geq 2)$ motions appear in low exciting frequency range, and longperiodic or chaotic motions can be observed between the existence regions of adjacent $1-p-p$ and $1-(p+1)-(p+1)$ motions. Regions of existence and stability of the impactless $1-0-0$, partial fundamental $1-p-p$ motions and complete chattering-impact motion with sticking can be obviously observed in the parameter plane of Fig. 2, which are identified by different colors, such as the impactless 1-0-0 motion is assigned to the red color, symmetric 1-1-1 motion to yellow, asymmetric 1-1-1 motion to brown and 1-2-2 motion to green, etc. It must be emphasized that the fundamental $1-p-p$ motions exhibit the properties of symmetry and asymmetry. The fundamental group of impact motions have the excitation period $(n=1)$ and differ by the number of impacts $p=0,1,2, \ldots, m \ldots$, i.e., $1-p-p$ impact motions. The practical significance is considerable that the $1-0-0$ impactless motion is included in this group, because the motion is subjected to similar transition phenomena as other $1-p-p$ impact motions. For enough large clearance, dynamic performance becomes relatively simple, and the system exhibits mainly impactless 1-0-0 or symmetric 1-1-1 motion in most regions of the parameter plane except small tongue-shaped regions nearby the critical line $L_{0}: R_{1-0-0} \cap R_{1-1-1}$ between adjacent regions of $1-0-0$ and symmetric $1-1-1$ motions; see Fig. 2. For slightly large $\delta$, the transition from $1-p-p$ to $1-(p+1)-(p+1)$ motion arises with decreasing the exciting frequency $\omega$ up to the grazing boundary $\mathrm{G}_{1-p-p}$; see Fig. 3(c). On the grazing boundary $G_{1-p-p}$, the mass block $M_{1}$ of the system, associated with $1-p-p$ motion, begins to touch the stops of the clearance. It can 
be regarded as the $1-(p+1)-(p+1)$ motion, however the impact velocity of the additional impact degenerates to touching. This means that a new periodic motion is characterized by $1-(p+1)-(p+1)$, in which the velocity of one of impacts increases gradually from zero with decreasing the exciting frequency $\omega$. Crossing the boundary $\mathrm{G}_{1-}$ $p-p, 1-(p+1)-(p+1)$ motion arises and the number of impacts of the periodic motion increases by two. Thus along the grazing boundary $\mathrm{G}_{1-p-p}$ both neighboring motions change continuously. A series of grazing bifurcations occur with decreasing the exciting frequency $\omega$ so that the number $p$ of impacts of the fundamental group of motions increases one by one at every rigid stop. As $p$ becomes big enough, the $1-p-p$ motion will exhibit the incomplete chattering-impact characteristics (i.e., a chattering sequence in an excitation period followed by a finite sequence of impacts with successively reduced velocity). The complete chattering-impact motion with sticking will occur with decreasing the exciting frequency $\omega$ up to the sliding bifurcation boundary. The lower the exciting frequency $\omega$ is, the more the number $p$ of impacts is, the longer the sticking time $t_{s}$ in the time interval of an exciting period is. Consequently, for slightly large $\delta$ the transition from 1-1-1 motion to complete chattering-impact motion is summarized as follows:

$\omega \downarrow: 1-\bar{p}-\bar{p}$

$\stackrel{\mathrm{S} B i f}{\leftarrow} \ldots \stackrel{\mathrm{G} B i f}{\leftarrow} 1-\tilde{p}-\tilde{p} \stackrel{\mathrm{G}}{\leftarrow \text { Bif }} \ldots \stackrel{\mathrm{GBif}}{\leftarrow} 1-(p+1)-(p+1) \stackrel{\mathrm{G}}{\leftarrow} \mathrm{Bif}_{1} 1-p-p \stackrel{\mathrm{G}}{\leftarrow \text { Bif }} \ldots$

G Bif $1-4-4 \stackrel{\text { G }}{\longleftarrow} 1-3 i f$

where let $1-\bar{p}-\bar{p}$ denote complete chatting-impact motion and $1-\tilde{p}-\tilde{p}$ represent incomplete chattering- impact motion, $S$ Bif and G Bif represent sliding and grazing bifurcations, respectively. This sticking chattering-impact vibration is periodic, which undergoes three processes in an exciting period in the following order: free flight, chattering-impact and sticking for the impacting mass. The periodically-forced impact system enters the sticking set via a chattering sequence followed by an infinite sequence of impacts with successively reduced velocity. Consequently, the impact mass of the system becomes constrained to the rigid stop in finite time $(t<2 \pi / \omega)$. This sequence is referred to as the complete chatteringimpact motion [33]. In contrast, incomplete chattering-impact motion occurs if the trajectory of the impact mass, after a finite number of impacts, reaches the non-sticking region [33]. It is important to note that chattering-impact vibration enlarges the adverse effects such as high noise levels and wear and so on.

The regular pattern of the transition, for slightly large $\delta$, is vividly plotted in the upper half plane of Fig. 3(c) and illustrated by grazing boundaries in Fig. 4(a). The analysis describes the system behavior on the boundaries of transition regions between $1-p-p$ and $1-(p+1)-(p+1)$ motions. For slightly large $\delta$, one can observe that the existence region of $1-p-p$ motion becomes gradually narrower and narrower with increase of the impact number of the fundamental periodic motion. This means that the existence regions of incomplete chattering-impact motions $(1-\tilde{p}-\tilde{p})$ are too narrow to be observed clearly. Consequently, $1-\tilde{p}-\tilde{p}$ motion can only be included in the blue region of $1-p-p(p>8)$ motions. However, it is important to note that the existence region of complete chattering-impact periodic motions is wider than that of $1-p-p$ motion $(p \geq 3)$ and corresponds to lower exciting frequencies, as seen in Fig. 3(c).

Period-doubling, saddle-node and pitchfork bifurcation boundaries are collectively referred to as stability boundaries for the convenience of the following analysis. Transition from complete to incomplete chattering-impact motion arises with increasing the exciting frequency $\omega$ up to the rising bifurcation boundary. Transition from $1-p-p$ to $1-(p-1)-(p-1)$ motion occurs with increasing the exciting frequency $\omega$ up to the saddle-node bifurcation boundary $\mathrm{SN}_{1-p-p}$. For slightly large $\delta$, the transition pattern from complete chattering-impact motion with sticking to 1-1-1 motion is summarized as follows:

$$
\begin{aligned}
& \omega \uparrow: 1-\bar{p}-\bar{p} \\
& \quad \stackrel{\mathrm{R}}{\rightarrow} \rightarrow 1-\tilde{p}-\tilde{p} \stackrel{\mathrm{SN} B i f}{\rightarrow} 1-(\tilde{p}-1)-(\tilde{p}-1) \\
& \quad \stackrel{\mathrm{SN} \text { Bif }}{\rightarrow} \mathrm{SN} \text { Bif } 1-p-p \stackrel{\mathrm{SN} \text { Bif }}{\rightarrow} 1-(p-1)-(p-1) \stackrel{\mathrm{SN} B i f}{\rightarrow} \ldots \\
& \quad \stackrel{\mathrm{SN} \text { Bif }}{\rightarrow} 1-3-3 \stackrel{\mathrm{SNBif}}{\rightarrow} 1-2-2 \stackrel{\mathrm{SNBif}}{\rightarrow} 1-1-1 .
\end{aligned}
$$

In Eq. (9), R Bif and SN Bif represent rising and saddle-node bifurcations, respectively.

Saddle-node bifurcation of $1-(p+1)-(p+1)$ motion corresponds to stability boundary $\mathrm{SN}_{1-(p+1)-(p+1)}$, on which two of impacts disappears and $1-p-p$ motion stabilizes after a jump transition. The transition between neighbor fundamental motions $1-p-p$ and $1-(p+1)-(p+1)$ is non-reversible except at the singular points of the alternate intersections of grazing and stability boundaries. Narrow hysteresis region exists between grazing bifurcation boundary $G_{1-p-p}$ of $1-p-p$ motion and saddle-node bifurcation boundary $\mathrm{SN}_{1-(p+)-(p+1)}$ of $1-(p+1)-(p+1)$ motion, as seen in Fig. 4(b). This means that both neighboring $1-p-p$ and $1-(p+1)-(p+1)$ motions are stable and can coexist in the dependence on the initial conditions of the system or on the way of the system parameters change in the hysteresis zones and they transit by a jump from one to the other on the grazing and saddle-node bifurcation boundaries of these regions. One neighboring motion switches to the other on the segments of the boundaries defining the hysteresis regions. Along the segment of grazing boundary, the
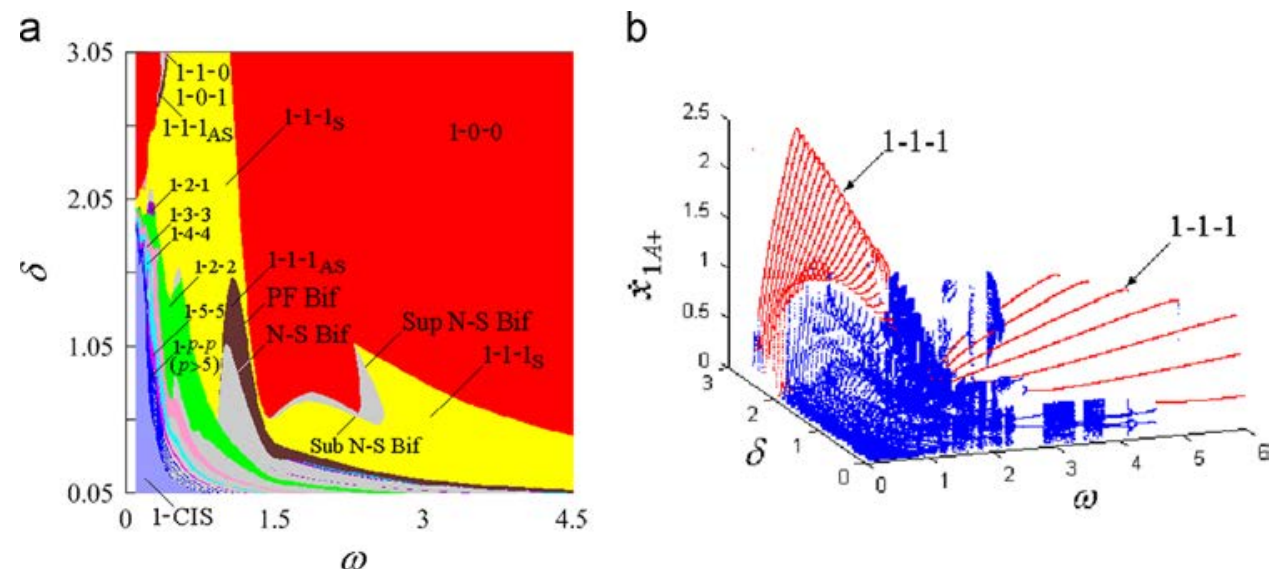

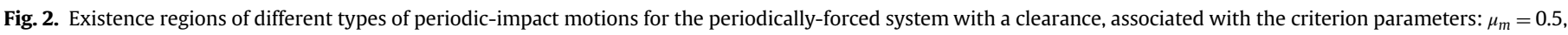

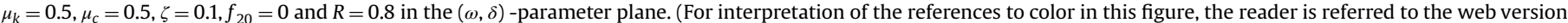
of this article.) 
a

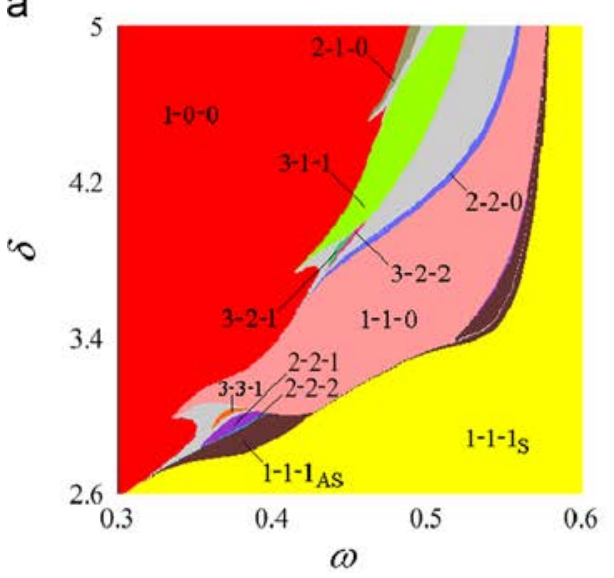

C

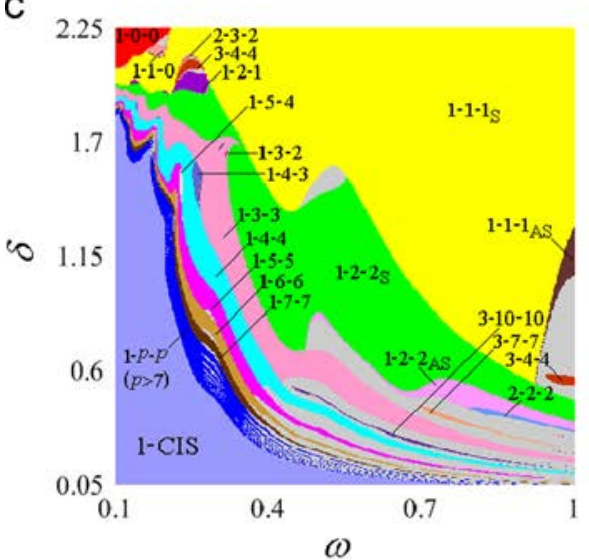

e

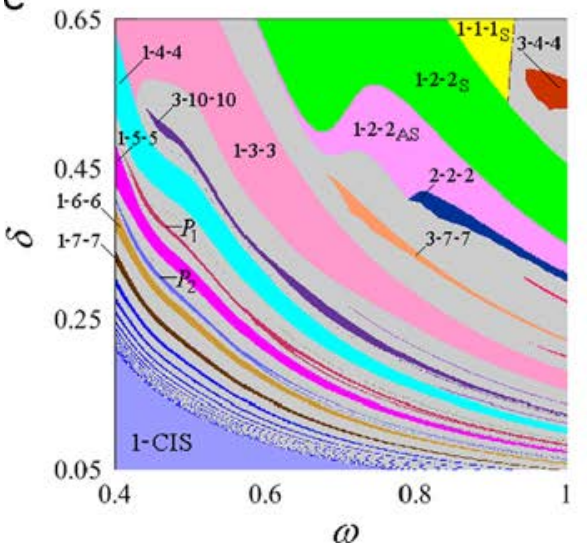

b

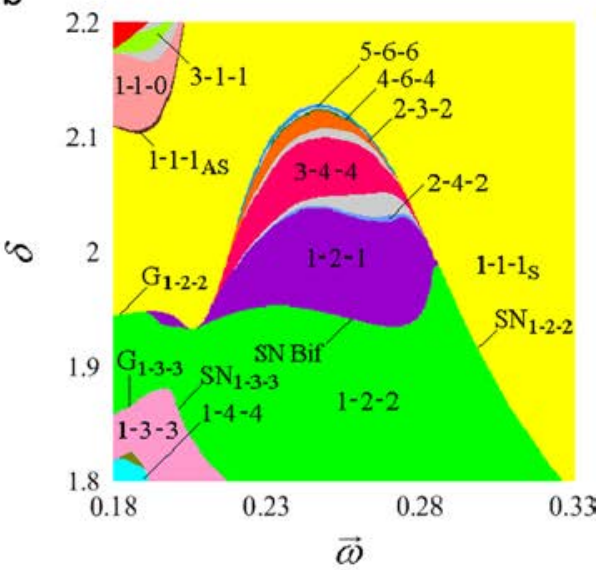

d

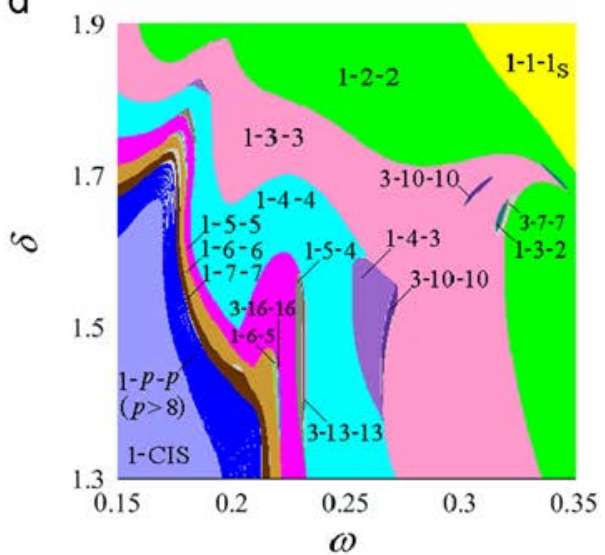

f

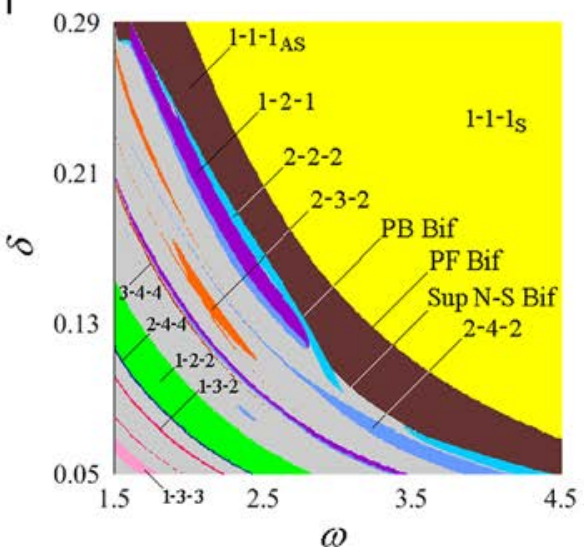

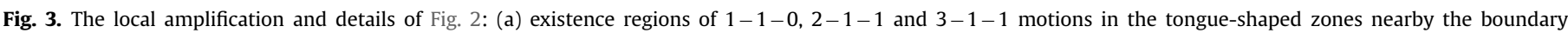

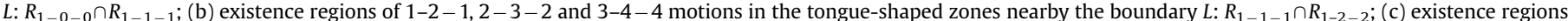

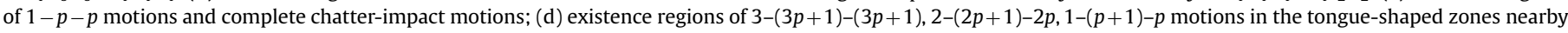

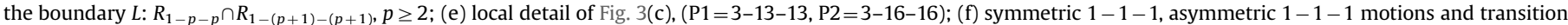
to $1-2-2$ motion.

impact number of a periodic motion increases by two. Conversely, along the other segment the impact number of the neighboring periodic motion decreases by two.

Moreover, for slightly large clearance $\delta$, there exist some small tongue-shaped regions nearby the critical line $L_{p}: R_{1-p-p} \cap$ $R_{1-(p+1)-(p+1)}$ between adjacent regions of $1-p-p$ and $1-(p+1)-(p+1)$ motions $(p \geq 1)$. Dynamics in tongue-shaped regions is more complex and emphatically introduced in the following context. With decreasing the clearance $\delta$, the region of $1-p-p$ motion approaches the grazing boundary, where the trajectory of $1-0-0$ impactless motion of the mass block $M_{1}$ or two additional impacts of $1-p-p$ motion trajectory begin to touch the stops. On the contrary, with increasing the clearance $\delta$, the region of $1-p-p$ motion reaches the stability boundaries associated with saddle-node bifurcation, crossing one of which $1-p-p$ motion loses its stability and transits to $1-(p-1)-(p-1)$ or $1-p-(p-1)$ motion. There exist a series of singular points $X_{i}$ on the boundaries between $1-p-p$ and $1-(p+1)-(p+1)$ motions, i.e., saddle-node bifurcation boundaries $\mathrm{SN}_{1-(p+1)-(p+1)}^{1-p-p}$ and $\mathrm{SN}_{1-(p+1)-(p+1)}^{1-(p+1)-p}$ of $1-(p+1)-(p+1)$ motion, bare and real grazing 
a

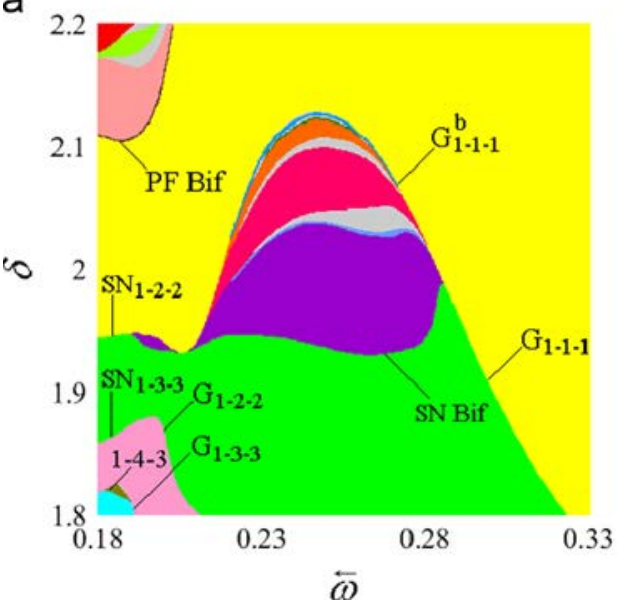

b

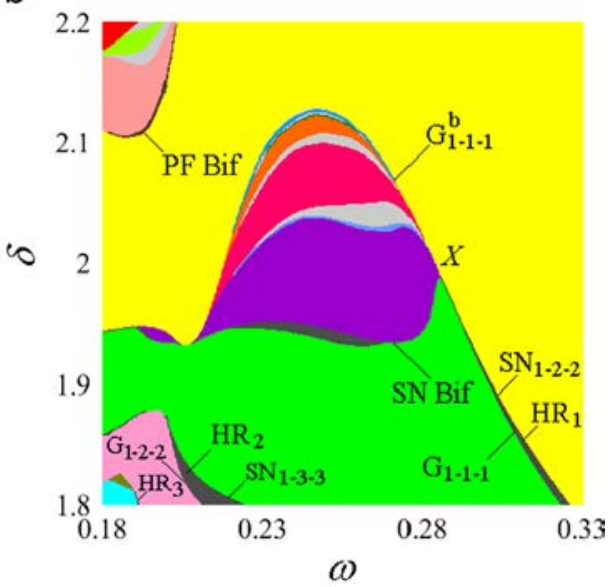

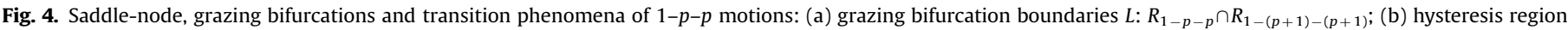
$\mathrm{HR}_{p}$ existing between grazing boundary $\mathrm{G}_{1-p-p}$ and saddle-node bifurcation boundary $\mathrm{SN}_{1-(p+1)-(p+1)}$.

boundaries $G_{1-p-p}$ and $G_{1-p-p}^{b}$ of $1-p-p$ motion alternately and mutually cross themselves at the points $X_{i}$ of intersection. It needs to be emphasized that the singular points of intersection of several bifurcation boundaries have a special significance and create inevitably two types of transition regions (hysteresis and tongueshaped regions). A special example is inserted to expound the above-mentioned analysis. A singular point $X$, the intersection of boundaries $\mathrm{SN}_{1-2-2}^{1-2-1}, \mathrm{SN}_{1-2-2}^{1-1-1}, \mathrm{G}_{1-1-1}$ and $G_{1-1-1}^{b}$, is described and illustrated in Fig. 4(b). Crossing the grazing boundary $\mathrm{G}_{1-1-1}$, the number of impacts increases by two and 1-1-1 motion transits to $1-2-2$ motion. This means that the grazing boundary $G_{1-1-1}$ is real. It is important to note that the grazing boundary $G_{1-1-1}^{b}$ is bare. Consequently, along $G_{1-1-1}^{b}$ the mass block $M_{1}$ of the system begins to touch the stops with almost zero before-impact velocity and 1-1-1 motion develops into a 1-2-2 motion which is unstable in the whole tongue-shaped region and the system transits to other type of impact motion by the unstable 1-2-2 motion. A tongue-shaped region is surrounded by the bare-grazing bifurcation boundary $G_{1-1-1}^{b}$ of 1-1-1 motion and saddle-node bifurcation boundary $\mathrm{SN}_{1-2-2}^{1-2}$ of $1-2-2$ motion, and corresponding hysteresis region is limited by real-grazing boundary $\mathrm{G}_{1-1-1}$ and saddle-node bifurcation boundary $\mathrm{SN}_{1-2-2}^{1-1-1}$. The grazing and saddle-node boundaries between the particular regions of $1-p-p$ and $1-(p+1)-(p+1)$ motions alternately intersect at singular points and so create two kinds of transient zones. There are partly zones of hysteresis and partly zones of tongue-shaped regions including fundamental $1-(p+1)-p$, subharmonic $3-(3 p+1)-$ $(3 p+1)$ and $2-(2 p+1)-2 p$ motions. The analysis for singular points $X$ can be extended to ascertain the limit of hysteresis and tongue-shaped regions. The hysteresis regions are limited at the top by the saddle-node bifurcation boundaries $\mathrm{SN}_{1-(p+1)-(p+1)}$ of $1-(p+1)-(p+1)$ motion and at the bottom by the real-grazing boundaries $\mathrm{G}_{1-p-p}$ of $1-p-p$ motion. The tongue-shaped regions are limited at the top by the bare-grazing bifurcation boundary $G_{1-p-p}^{b}$ of $1-p-p$ motion and at the bottom by the saddle-node bifurcation boundary $\mathrm{SN}_{1-(p+1)-(p+1)}^{1-(p+1)-p}$ of $1-(p+1)-(p+1)$ motion. A series of zones of fundamental and subharmonic impact motions, such as $1-(p+1)-p, 2-(2 p+1)-2 p$ and $3-(3 p+1)-$ $(3 p+1)$ impact orbits, etc., are found to occur in the tongueshaped regions. Existence region of a periodic or subharmonic impact motion, in the parameter plane, is called parameter island of the corresponding motion. The phenomena can be observed in Fig. 3(c). Details of Fig. 3(c), associated with the tongueshaped regions and subharmonic impact characters, are shown, respectively, in Fig. 3(b) and (d). The positions of the corresponding parameter islands of $1-(p+1)-p, 3-(3 p+1)-$ $(3 p+1)$ and $2-(2 p+1)-2 p$ motions arrange from the bottom up (or from left to right) in this order in the tongue-shaped region, and the area of the parameter islands becomes smaller and smaller in this order. It is important to note that no motion with the exciting force period and symmetric impacts (i.e., symmetric $1-p-p$ motion) is stable or can exist in the tongue-shaped regions. Consequently, $1-(p+1)-p, 2-(2 p+1)-2 p$ and $3-(3 p+1)-$ $(3 p+1)$ motions and chaotic ones dominate in the regions. The continuous transition between neighboring $1-p-p$ and $1-(p+1)-$ $(p+1)$ motions can exist only at the singular points $X$ of the intersections of grazing and saddle-node boundaries $\mathrm{G}_{1-p-p}$, $G_{1-p-p}^{b}$ and $\mathrm{SN}_{1-(p+1)-(p+1)}$. The motions which the system exhibits at the singular points $X_{i}$ are identical. The $1-p-p$ motion, associated with the new-appeared periodic touching the stops of the clearance, can be regarded as that the certain two periodic impacts of the $1-(p+1)-(p+1)$ motion degenerates into the touching. Consequently, $1-p-p$ motion will end on its grazing boundary and $1-(p+1)-(p+1)$ motion will emerge. In contrast, the adverse transition from one neighboring fundamental motion to the other, at the singular points $X_{i}$, is also similar to that abovementioned.

A tongue-shaped region nearby the critical line $L_{0}: R_{1-0-0} \cap$ $R_{1-1-1}$ between adjacent regions of 1-0-0 and 1-1-1 motions are shown in Fig. 3(a). The impact motion is invariably found below the grazing boundary $G_{1-0-0}$. The regions of the kinds of impact motions ascertained are marked by different colors and corresponding symbol $n-p-q$. The tongue-shaped region is limited at the bottom by the pitchfork bifurcation boundary $\mathrm{PF}_{1-1-1}$ of symmetric 1-1-1 motion and at the top by the bare-grazing bifurcation boundary $G_{1-0-0}^{b}$ of the impactless motion. Increasing the exciting frequency $\omega$ (or decreasing the clearance $\delta$ ) up to the boundary $L_{\mathrm{G}}: R_{1-0-0} \cap R_{\mathrm{T} 1-0-0}$, the mass block $M_{1}$ of the system, associated with 1-0-0 impactless forced vibration, begins to touch the stops with almost zero impact velocities, i.e., the bare touching. On the boundary $L_{\mathrm{G}}: R_{1-0-0} \cap R_{\mathrm{T} 1-0-0}$, the impactless motion develops into a 1-1-1 motion which is unstable in the whole tongue-shaped region and the system transits to other type of impact motion by the unstable 1-1-1 motion. In the tongueshaped region $R_{\mathrm{T} 1-0-0}$ no motion with the exciting force period and symmetric impacts is stable or can exist so that 1-1-0 (or 1-0-1), 2-1-0 (or 2-0-1) and 3-1-1 motions, 2-2-1 (or 2-1-2), $3-3-1$ (or 3-1-3), 3-2-1 (or 3-1-2), 3-2-2 motions and chaotic motions dominate. By means of a theoretical or numerical analysis it is possible to find this zone of the existence and stability 
subregions of $1-1-0,2-1-0$ and symmetric $3-1-1$ motions. The zones of 1-1-0, 2-1-0 and symmetric 3-1-1 motions are limited from above by stability boundaries $\mathrm{PD}_{1-1-0}, \mathrm{SN}_{2-1-0}$ and $\mathrm{PF}_{3-1-1}$ and from down by grazing boundaries $\mathrm{G}_{1-1-0}, \mathrm{G}_{2-1-0}$ and $G_{3-1-1}$ which probably correspond to stable or unstable grazing bifurcations, respectively. Partial subregions are drawn in Fig. 3(a) and surrounded by the stability and grazing boundaries. With increasing the exciting frequency $\omega$ up to crossing the boundary $L_{\mathrm{PF}}: \quad R_{1-1-1} \cap R_{\mathrm{T} 1-0-0}$, symmetric 1-1-1 motion stabilizes. And then the symmetric 1-1-1 motion transits by jump to the impactless motion on the stability boundary $L_{\mathrm{SN}}: R_{1-1-1}$ $\cap R_{1-0-0}$, where the differences $\Delta X^{(i)}=\left(\Delta \tau, \quad \Delta x_{2}^{(i)}, \Delta \dot{x}_{1+}^{(i)}, \quad \Delta \dot{x}_{2}^{(i)}\right)^{\mathrm{T}}$ of the fixed point $X^{*}=\left(\tau_{0}, x_{20}, \dot{x}_{1+}, \dot{x}_{20}\right)^{\mathrm{T}}$ of symmetric 1-1-1 motion in Poincaré section $\sigma_{p}$ grow with the same sign and the impact finally vanishes and 1-0-0 motion stabilizes. As a result, beyond the boundary $L_{S N}: R_{1-1-1} \cap R_{1-0-0}$ no solution of symmetric 1-1-1 motion is real; the saddle-node bifurcation of the motion corresponds to the state along the stability boundary.

With $\omega$ increasing and crossing a tongue-shaped zone between adjacent regions of 1-0-0 and 1-1-1 motions, a most complex transition, associated with a horizontal scan for certain fixed value of clearance (or a vertical scan for certain fixed value of exciting frequency), is summarized as follows:

$\omega \uparrow$ or $\delta \downarrow: 1-0-0 \rightarrow$ Unstable G Bif $\rightarrow \ldots \rightarrow 2-1-0 \rightarrow \mathrm{G}$ Bif $\rightarrow \ldots \rightarrow 3-1-1_{A S} \rightarrow$ Inverse Pitchfork Bif $\rightarrow 3-1-1_{S} \rightarrow \mathrm{G}$ Bif $\rightarrow \ldots \rightarrow 2-2-0 \rightarrow 1-1-0 \rightarrow \mathrm{G}$ Bif $\rightarrow$ $1-1-1_{A S} \rightarrow$ Inverse Pitchfork Bif $\rightarrow 1-1-1_{S}$,

where G Bif is probably stable or unstable grazing bifurcation. The unstable grazing bifurcation causes long-periodic multi-impact or chaotic motion due to the bare touching of the mass block $M_{1}$ with the stops. Motion amplitude $x_{1 m p}(\omega)$ and impact velocity $\dot{x}_{1 A-}(\omega)$ associated with a transition crossing a tongue-shaped zone between adjacent regions of 1-0-0 and 1-1-1 motions, along a horizontal scan for $\delta=5.0$, are shown in Fig. 5. During a horizontal scan of change of the value of exciting frequency through regions of different types of impact motions, the diversity and evolution of the system motion can be observed by the bifurcation diagrams shown in Fig. 5(a) and (b). It is important to note that the bifurcation diagram $x_{1 m p}(\omega)$ corresponds to the Poincaré section $\sigma_{n}=\left\{\left(x_{1}, \dot{x}_{1}, x_{2}, \dot{x}_{2}, t\right) \in \mathbf{R}^{4} \times \mathbf{S}, \quad x_{1}=x_{1} \min \bmod (t=2 \pi / \omega)\right\}$, which represents the minimum displacements of mass $M_{1}$ appearing in every excitation period $T_{1}=2 \pi / \omega$. The bifurcation diagram $\dot{x}_{1 A-}(\omega)$ is related to the impact Poincaré section $\sigma_{p}=\left\{\left(x_{1}, \dot{x}_{1}, x_{2}, \dot{x}_{2}, t\right) \in\right.$ $\left.\mathbf{R}^{4} \times \mathbf{S}, x_{1}=\delta, \quad \dot{x}_{1}=\dot{x}_{1 A-}\right\}$, which corresponds to the beforeimpact velocity of every impact. As a result, it is convenient to ascertain $n-p-q$ motion according to the number $p$ of branches of $\dot{x}_{1 A_{-}}(\omega)$ and the number $n$ of branches of $x_{1 m p}(\omega)$ of a certain impact motion. The windows of $2-1-0$ (or $2-0-1$ ), 3-1-1 and 1-1-0 (or 1-0-1) motions are easily observed by Fig. 5. Perioddoubling bifurcation of 1-1-0 (or 1-0-1) motion occurs with decreasing the exciting frequency $\omega$. However we can note that existence region of the period-doubling orbit is too small to be observed; see Fig. 6 (the detail of Fig. 5(b)). Long-periodic multiimpact or irregular impact motions arise successively via baregrazing bifurcations of 2-1-0 (2-1-0) and asymmetric 3-1-1 motions with increasing the exciting frequency $\omega$. A representative 3-1-1 motion is shown for $\omega=0.51$ in Fig. 7(a). A bare-grazing contact instability of 3-1-1 motion occurs at $\omega=0.525659$. The transition to chaos via the bare-grazing bifurcation of the motion is depicted by the phase plane portraits shown in Fig. 7(a)-(c).

The zones of tongue-shaped regions between adjacent regions of $1-p-p$ and $1-(p+1)-(p+1)$ motions are shown, respectively, in Fig. $3(\mathrm{~b})$ and (d). Only the zones of the existence and stability subregions of $1-(p+1)-p, 3-(3 p+1)-(3 p+1)$ and $2-(2 p+1)-2 p$ motions are provided in the tongue-shaped regions. The zones of some impact motions caused by correlative bifurcations of the three motions are too small to be observed clearly, so these motions and other motions not ascertained in the tongue-shaped regions are all marked by gray color. Part zones of $1-(p+1)-p$, $3-(3 p+1)-(3 p+1)$ and $2-(2 p+1)-2 p$ motions are drawn in Fig. 3 (c), the details of which are shown by the next enlarged subregions in Fig. 3(b) and (d). The zones of $1-(p+1)-p, 2-(2 p+1)-2 p$ and $3-(3 p+1)-(3 p+1)$ motions are limited from above in turn by period doubling bifurcation boundaries $\mathrm{PD}_{1-(p+1)-p}, \mathrm{PD}_{2-(2 p+1)-}$ $2 p$ and $\mathrm{PD}_{3-(3 p+1)-(3 p+1)}$ and from down in turn by saddle-node bifurcation boundary $\mathrm{SN}_{1-(p+1)-p}$, grazing bifurcation boundaries $\mathrm{G}_{2-(2 p+1)-2 p}$ and $\mathrm{G}_{3-(3 p+1)-(3 p+1)}$, respectively. With $\omega$ or $\delta$ decreasing and crossing a tongue-shaped zone between adjacent regions of $1-p-p$ and $1-(p+1)-(p+1)$ motions $(p \geq 1)$, a most complex transition, associated with a horizontal scan for certain fixed value of clearance (or a vertical scan for certain fixed value of exciting frequency), is summarized as follows:

$$
\begin{aligned}
& \omega \downarrow \text { or } \delta \downarrow: 1-(p+1)-(p+1)_{S} \leftarrow \text { SN Bif } \leftarrow 1-(p+1)-p \leftarrow 2-(2 p+2)-2 p \\
& \leftarrow \ldots \leftarrow \text { G Bif } \leftarrow 3-(3 p+1)-(3 p+1) \leftarrow 6-(6 p+2)-(6 p+2) \\
& \leftarrow \ldots \leftarrow \text { G Bif } \leftarrow 2-(2 p+1)-2 p \leftarrow 4-(4 p+2)-4 p \leftarrow \ldots \leftarrow \text { unstable G Bif } \leftarrow 1-p-p_{S},
\end{aligned}
$$

where $\mathrm{G}$ Bif is probably real or bare unstable grazing bifurcation. Motion amplitude $x_{1 m p}(\delta)$ and impact velocity $\dot{x}_{1 A-}(\delta)$ associated with a transition crossing a tongue-shaped zone between adjacent regions of $1-1-1$ and $1-2-2$ motions, along a vertical scan for $\omega=0.26$, are shown in Fig. 8. The windows of $1-2-1,3-4-4$ and 2-3-2 motions are easily found by analyzing Fig. 8(a) and (b).

\section{b}

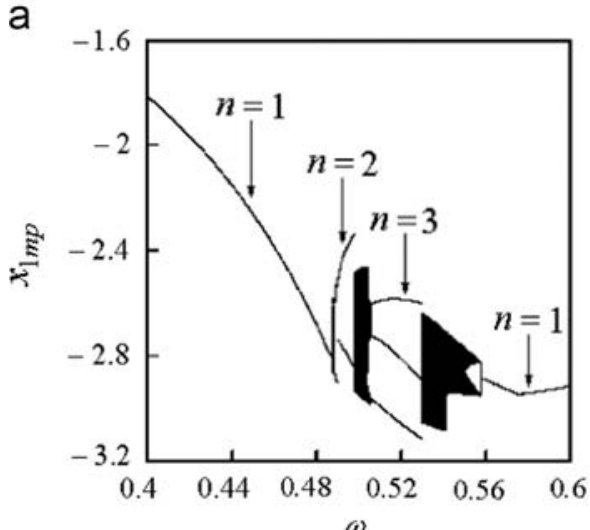

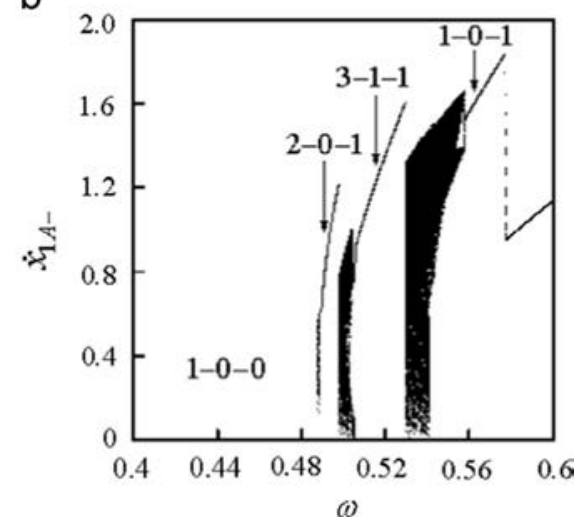

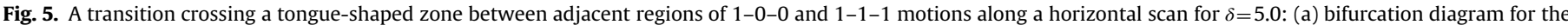
lowest displacement $x_{1 m p}(\omega)$ of the motion appearing in every period $T$; (b) bifurcation diagram for impact velocity $\dot{x}_{1 A-}(\omega)$. 

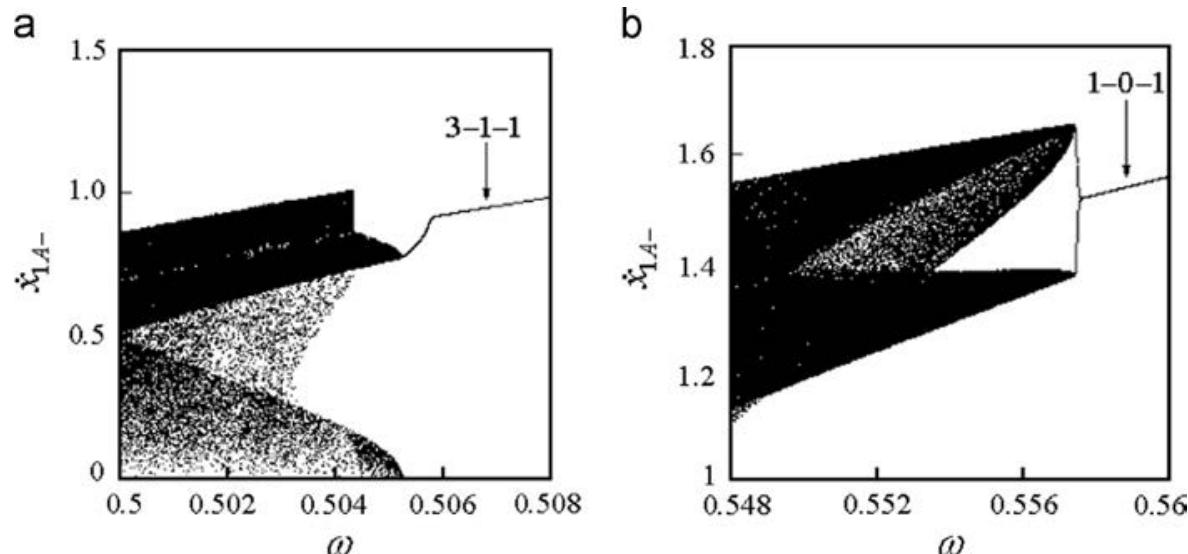

Fig. 6. Details of Fig. 5(b).
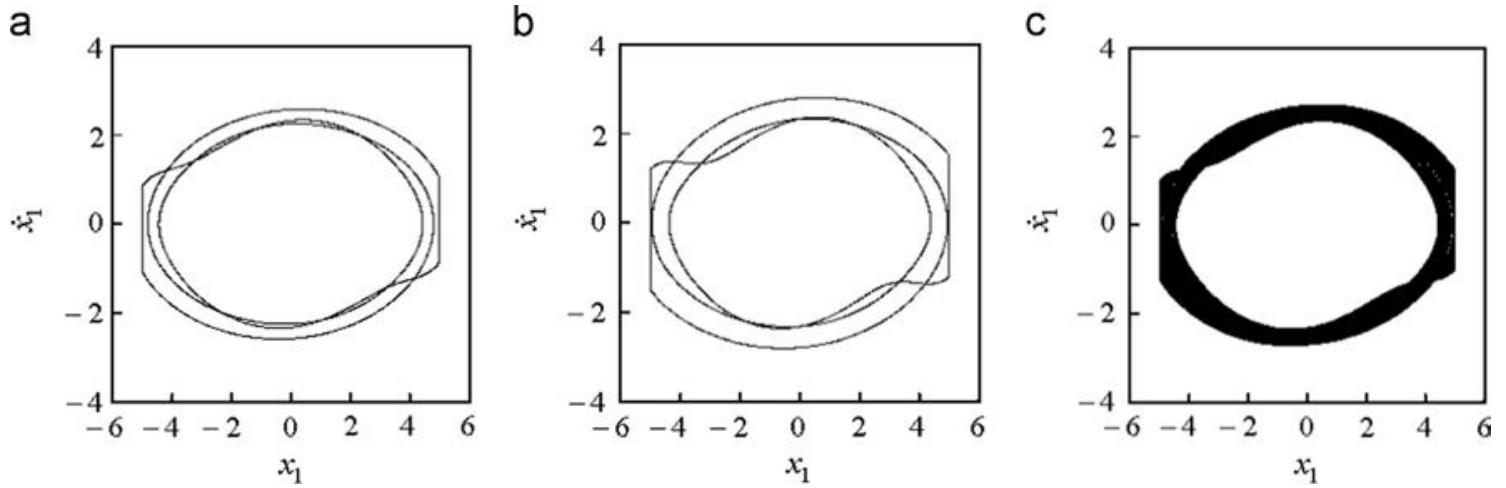

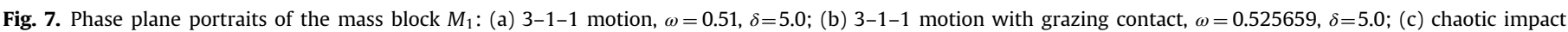
motion, $\omega=0.5256589, \delta=5.0$.
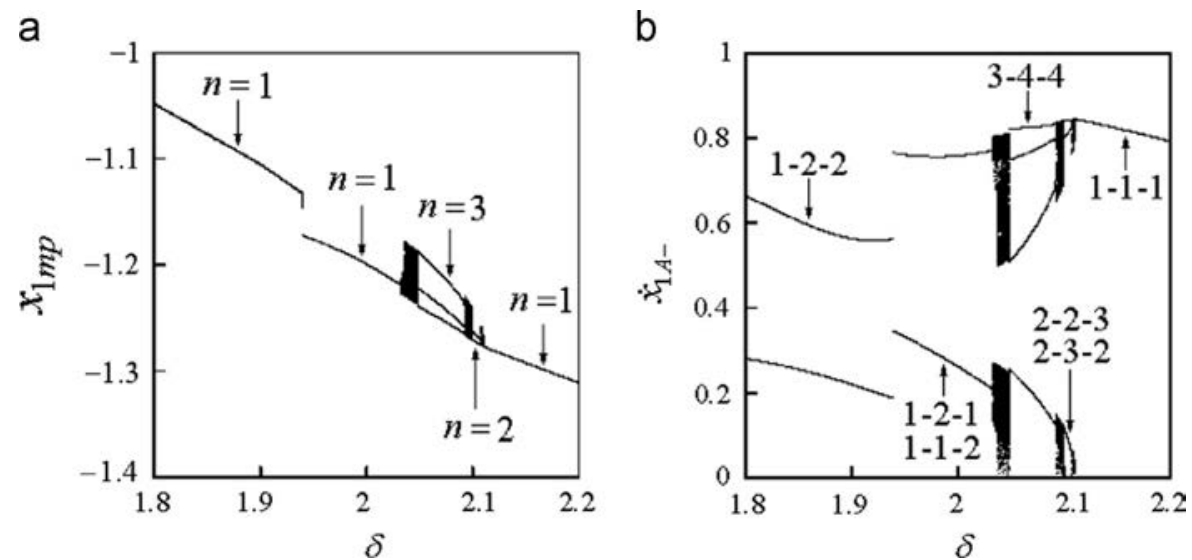

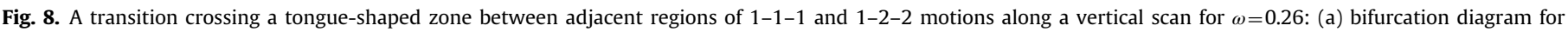
$x_{1 m p}(\delta)$; (b) bifurcation diagram for impact velocity $\dot{x}_{1 A_{-}}(\delta)$.

Period-doubling bifurcations of 1-2-1, 3-4-4 and 2-3-2 motions occur successively with increasing the clearance $\delta$. Existence regions of $2-4-2,6-8-8$ and $4-6-4$ motions are too narrow to be observed in Fig. 8. However they can be observed by Fig. 9 (details of Fig. 8(b)). Long-periodic multi-impact or chaotic motions arise successively due to the grazing bifurcation of 3-44 and 2-3-2 motions with decreasing the clearance $\delta$; see Fig. 8. A bare-grazing contact instability of 3-4-4 motion occurs at $\delta=2.047421$. Consequently, the unstable grazing bifurcation of the motion leads to irregular impact motion immediately, which is depicted in Fig. 10.

For very small clearance, the transition from $1-p-p$ to $1-(p+1)-(p+1)$ motion $(p \geq 2)$ is far more complicated than that associated with large clearance, which basically goes through three processes. First, pitchfork bifurcation of symmetric $1-p-p$ motion occurs with decreasing $\omega$. Consequently, asymmetric $1-p-p$ motion stabilizes, two antisymmetric forms of which can exist in the dependence on the initial conditions of the system. Secondly, period-doubling bifurcation of asymmetric $1-p-p$ motion occurs and it undergoes a succession of period-doubling bifurcations with decrease in $\omega$, which eventually result in apparently non-periodic or chaotic motions. Finally, symmetric $1-(p+1)-(p+1)$ motion is generated by a degeneration of chaos with decreasing $\omega$. Moreover, there exists the zone of $3-(p+1)-$ $(p+1)$ motion in the transition region from $1-p-p$ to $1-(p+1)-$ $(p+1)$ motion, which are observed in Fig. 3(e) and (f). For small 
a

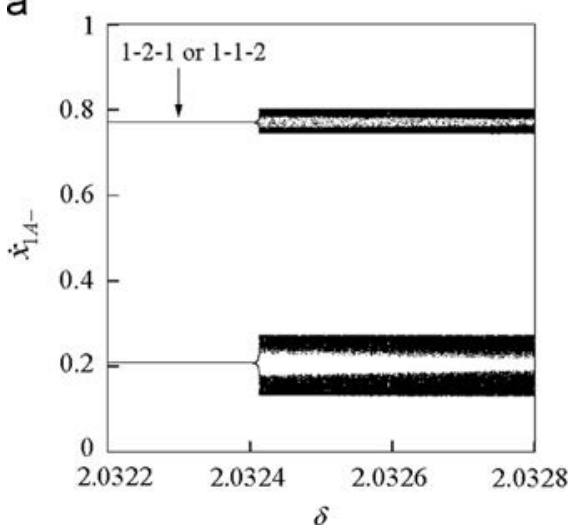

b

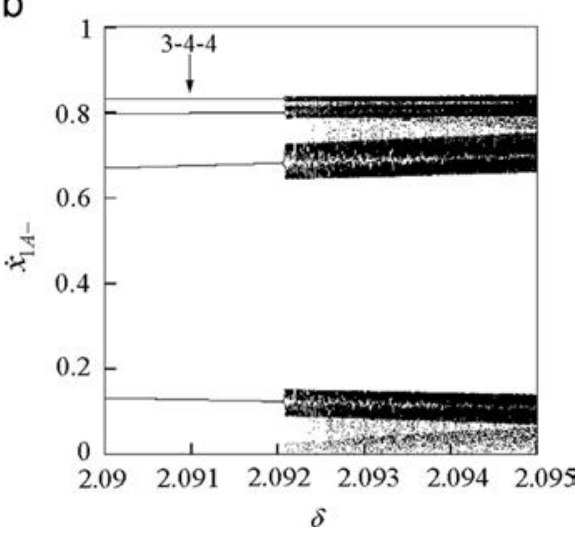

Fig. 9. Details of Fig. 8(b).

a

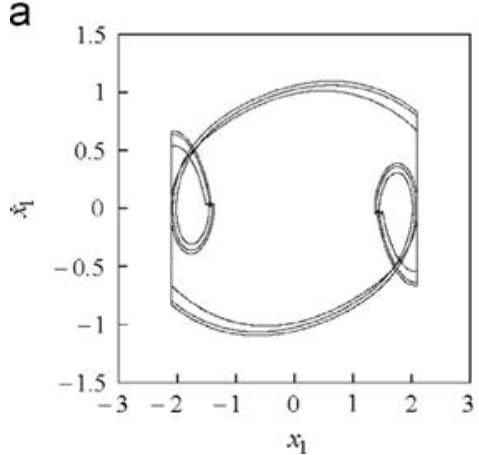

b

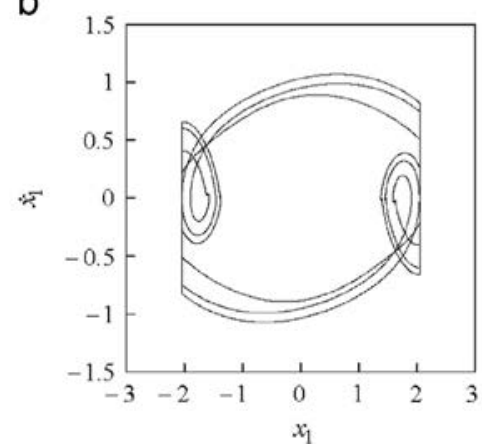

C

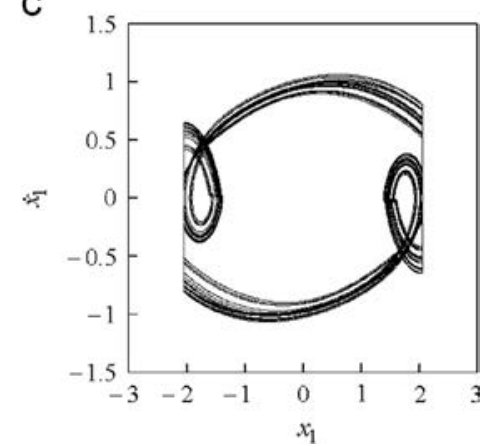

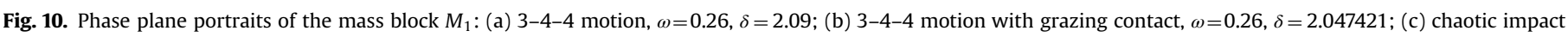
motion, $\omega=0.26, \delta=2.047420$.

clearance, another transition from asymmetric 1-1-1 to symmetric 1-2-2 motion may also occur. First, Neimark-Sacker bifurcation of asymmetric 1-1-1 motion occurs with decreasing $\omega$ so that quasi-periodic impact motions arise; secondly, the quasiperiodic impact motion transits to chaos via phase locking; finally, symmetric 1-2-2 motion is generated by a degeneration of chaos with decrease in $\omega$. The transition from $1-p-p$ to $1-(p+1)-(p+1)$ motion $(p \geq 2)$ can be observed in Fig. 3(e) and Fig. 11.

For very small clearance, symmetric 1-1-1 motion first goes through pitchfork bifurcation with decreasing $\omega$. Secondly, perioddoubling bifurcation of asymmetric 1-1-1 motion occurs; perhaps a succession of period-doubling bifurcations occur and eventually result in apparently non-periodic or chaotic motions with decreasing $\omega$. Finally, symmetric $1-2-2$ motion is generated by a degeneration of chaos. Moreover, there exist the zones of 1-2-1, $2-3-2,3-4-4$ and $2-4-2$ motions in the transition region from asymmetric $1-1-1$ to symmetric $1-2-2$ motion. Based on the bottom of Fig. 3(f), the whole transition can be observed.

Period doubling bifurcation of asymmetric 1-1-1 motion basically corresponds to very small clearance. However, it is possible to occur Neimark-Sacker bifurcation of asymmetric 1-1-1 motion in certain an interval associated with very small clearances, as seen in Fig. 3(f). For slightly large clearance, Neimark-Sacker bifurcation of symmetric 1-1-1 motion occurs in high exciting frequency range and quasi-periodic impact motions transit to chaotic impact motions via phase locking or torus doubling, and impactless $1-0-0$ motion is finally generated by a degeneration of chaos with decrease in $\omega$; Neimark-Sacker bifurcation of asymmetric 1-1-1 motion generally corresponds to slightly low exciting frequencies, as seen in Fig. 2(a). Representative bifurcation diagrams of before-impact velocity as a function of the exciting frequency are shown for $\delta=0.1,0.3,0.545,0.6,0.8$ and
1.1 in Fig. 11(a)-(f), which illustrate bifurcation characteristics of symmetric 1-1-1 motion.

\section{The correlative relationship between dynamic performance and system parameters}

It is important to analyze the detailed influence of each parameter on dynamics of the system to reveal the correlative relationship and matching law between dynamic performance and system parameters. In the following text, we will discuss the influence of each parameter on dynamics of the system by comparing with dynamics associated with the criterion parameters. In Fig. 12 are plotted the existence regions of different types of periodic-impact motions for the non-smooth vibratory system with variation of the mass distribution $\mu_{m}$. The selected values of parameter $\mu_{m}$ are given in graphical interpretation of Fig. 12, the other parameters are the same as the remainder of the criterion parameters. For the following analyses associated with the selected parameters $\mu_{k}, \mu_{c}, \zeta, f_{20}$, and $R$, the graphical interpretations are similar to that of Fig. 12. The effects of changes in $\mu_{m}$ are emphatically analyzed by selecting its different values and scanning the exciting frequency $\omega$ and clearance $\delta$. As the mass distribution $\mu_{m}$ becomes small enough, the case corresponds to high mass concentration on the impacting mass block, the system is actually equivalent to the mechanical model that the mass block $M_{1}$ is connected to the supporting base by the linear springs $K_{1}$ and $K_{2}$ in series and linear viscous dashpots $C_{1}$ and $C_{2}$ in series and behaves as a single-degree-of-freedom impact oscillator. The increase of mass parameter $\mu_{m}$ brings great changes in the distribution and existence region of symmetric 1-1-1 motion of the system. With increasing $\mu_{m}$ in the interval $\mu_{m} \in[0.05,0.5]$, one can find that 

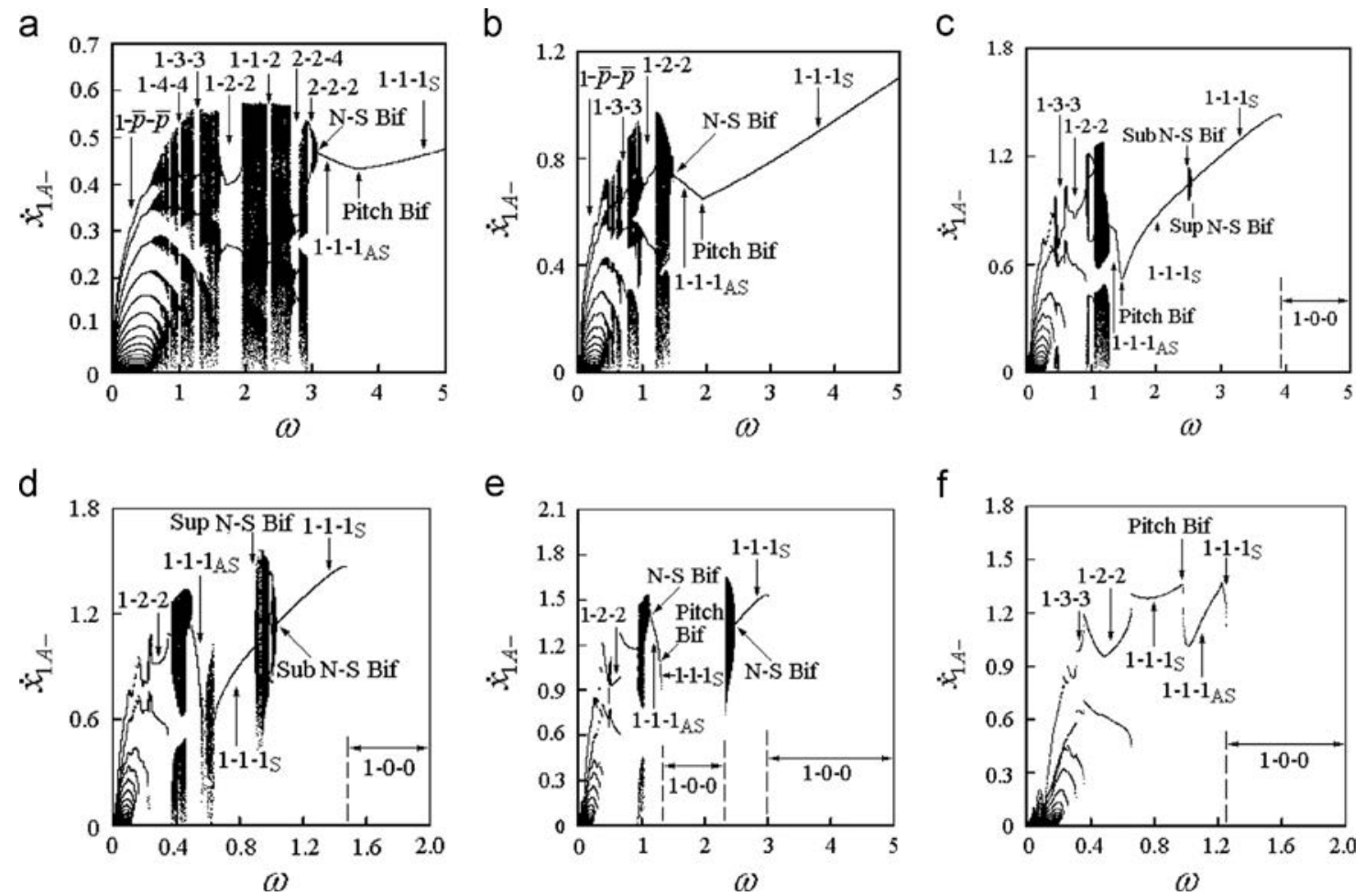

Fig. 11. Bifurcation diagrams of impact velocity as a function of the exciting frequency $\dot{x}_{1 A_{-}}$: (a) $\delta=0.1$; (b) $\delta=0.3$; (c) $\delta=0.545$; (d) $\delta=0.6$; (e) $\delta=0.8$; (f) $\delta=1.1$.
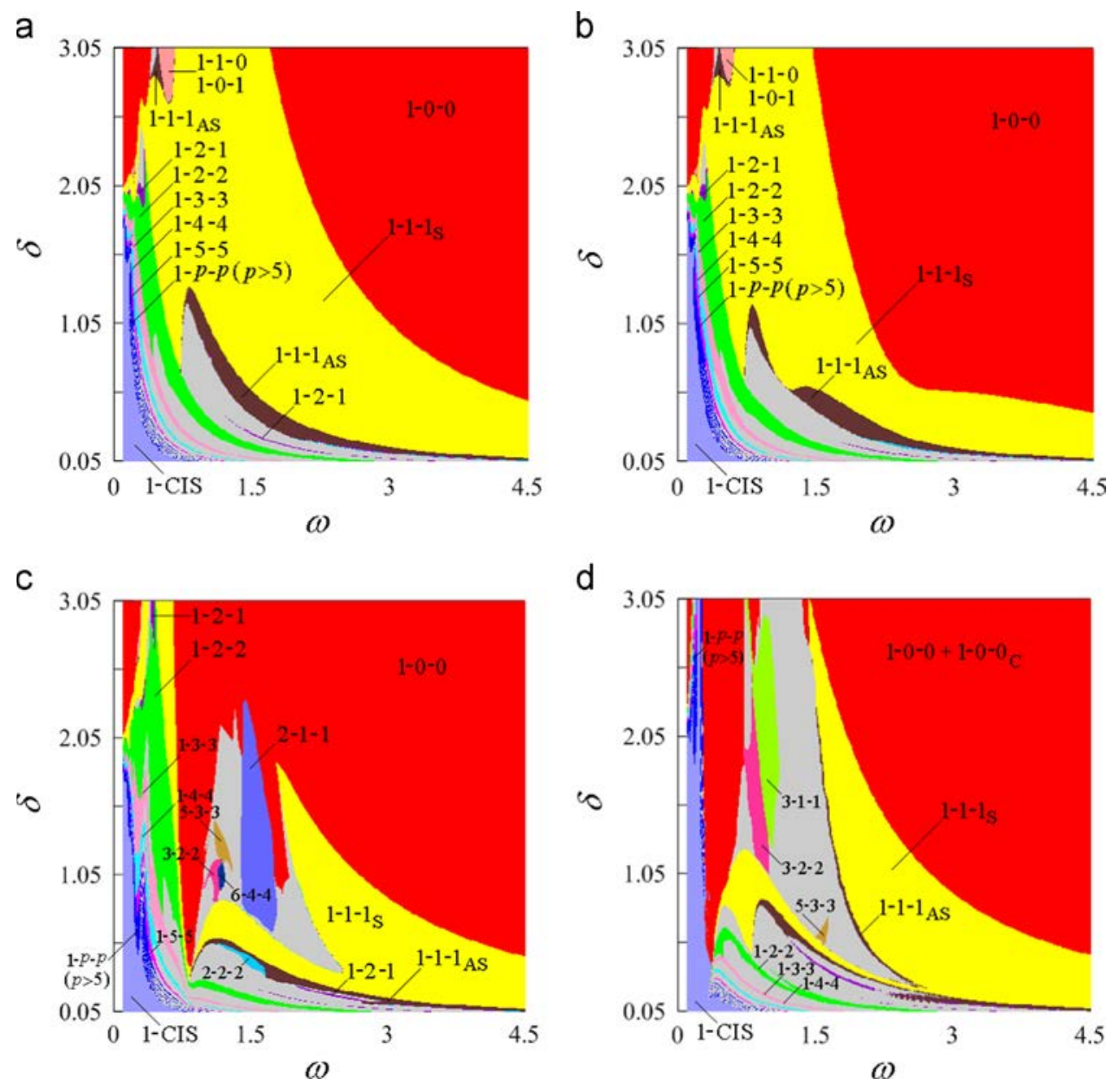

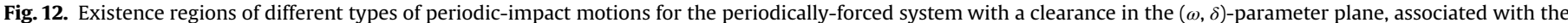

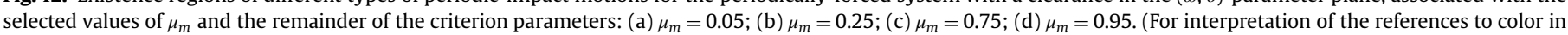
this figure, the reader is referred to the web version of this article.) 
a

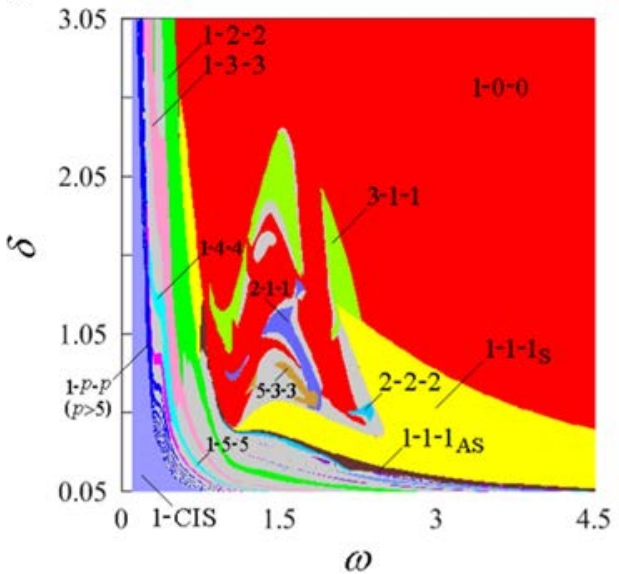

C

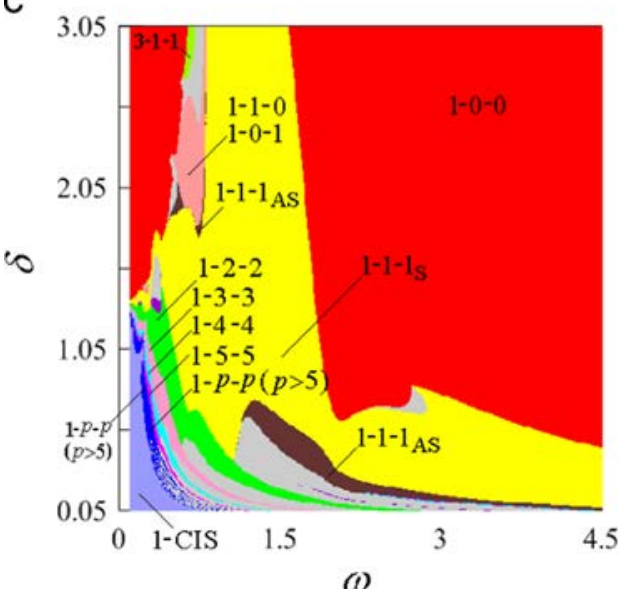

b

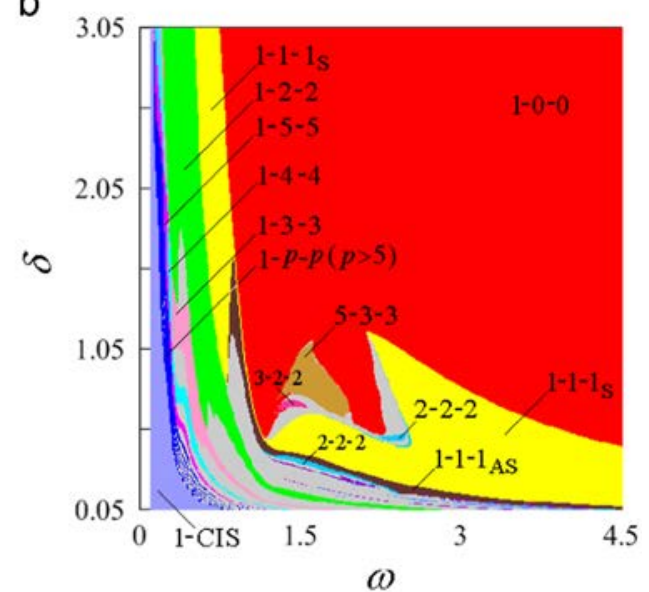

d

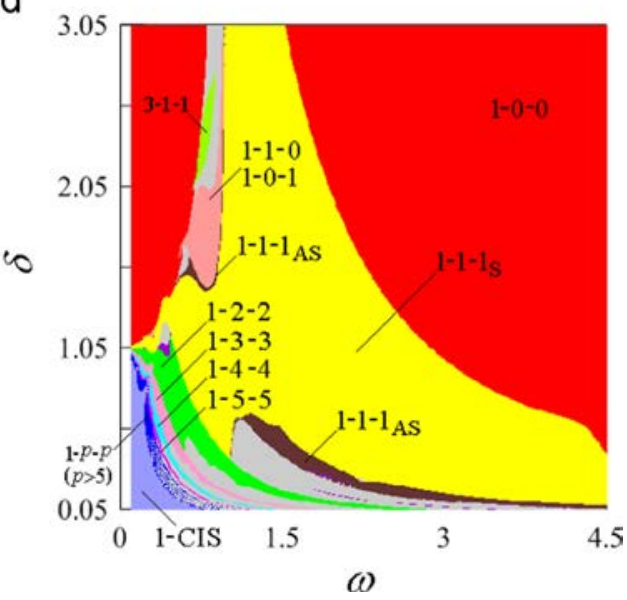

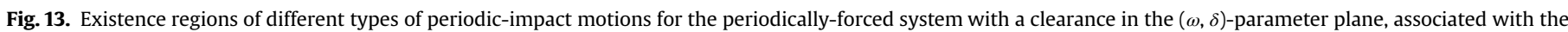
selected values of $\mu_{k}$ and the remainder of the criterion parameters: (a) $\mu_{k}=0.05$; (b) $\mu_{k}=0.25$; (c) $\mu_{k}=0.75$; (d) $\mu_{k}=0.95$.

the existence region of symmetric 1-1-1 motion of the system shrinks remarkably, as seen in Fig. 2(a), Fig. 12(a) and (b). The existence region of symmetric 1-1-1 motion is almost divided into two parts with $\mu_{m}$ increasing progressively and crossing $\mu_{m}=0.67$, and its left part shrinks gradually. Correspondingly, its right part expands gradually. The system exhibits complex dynamical characteristics between divided existence regions of symmetric 1-1-1 motion, impactless, subharmonic impact (2-1-1 and 3-1-1, etc.), long-periodic multi-impact, quasi-periodic impact and chaotic motions dominate. Moreover, the existence regions of $1-p-p$ motions $(p \geq 2)$ shrink slightly at the outset to the lower bound and after that begin to enlarge with increase in $\mu_{m}$. However the existence region of complete chattering-impact motions always keep slight extension with increasing the mass distribution $\mu_{m}$. As $\mu_{m}$ becomes very large, this means that high mass concentration on the non-impacting mass block. In this case the system is actually equivalent to one in which the exciting force $P_{1} \sin (\Omega T+\tau)$ directly acts on the right end of the linear springs $K_{1}$ and linear viscous dashpots $C_{1}$. The system exhibits more complex dynamical characteristics in the extreme case. Consequently, more types of periodic-impact motions arise and the existence region of complete chattering-impact motions with sticking is extended to larger clearance interval. It is possible for more complex sequences of subharmonic impact motions to occur in large $\mu_{m}$ case, such as 2-11, 3-1-1, 3-2-2 and 5-3-3, etc., which can be observed in Fig. 12 (c) and (d). The regions of periodic-impact motions unascertained, quasi-periodic impact motions and chaotic impact motions are marked by blue color. We can note that these regions are very large in the $(\omega, \delta)$-parameter plane as $\mu_{m}$ is large enough; see Fig. 12(d). The phenomenon can be explained by three reasons. Firstly, the existence regions of some periodic-impact motions ascertained are too small to be observed clearly; secondly, the number $n$ of excitation period or the number $p$ of impacts in the whole motion period is too big for the corresponding periodic-impact motions to be ascertained effectively; finally, the remainder are basically quasiperiodic and chaotic motions. The larger the mass distribution $\mu_{m}$ is, the more complex dynamics of the system is.

Existence regions of different types of periodic-impact motions for the non-smooth vibratory system with variation of the stiffness distribution $\mu_{k}$ are shown in Fig. 13. The effects of changes in $\mu_{k}$ are analyzed by comparing with the results associated with the criterion parameters. As the stiffness distribution $\mu_{k}$ becomes very small, the stiffness $K_{1}$ connecting two mass blocks is much larger than the stiffness $K_{2}$ connecting the mass block $M_{2}$ and the supporting base so that the difference between the displacements of two mass blocks is very small. The system exhibits more complex dynamical characteristics in the extreme case associated with very small $\mu_{k}$. Consequently, more types of periodic-impact motions arise and the existence regions of $1-p-p$ motions $(p \geq 2)$ are extended to larger clearance interval. Many complex sequences of subharmonic impact motions, such as $2-1-1,3-1-1,3-2-2$ and $5-3-3$, etc., are found to exist in small $\mu_{k}$ case, as observed in Fig. 13(a) and (b). As $\mu_{k}$ is small enough, the existence region of symmetric 1-1-1 motion is almost divided into two parts, and its left part expands gradually and the right part slightly shrinks with increasing $\mu_{k}$ progressively. Moreover, for small $\mu_{k}$ the existence 

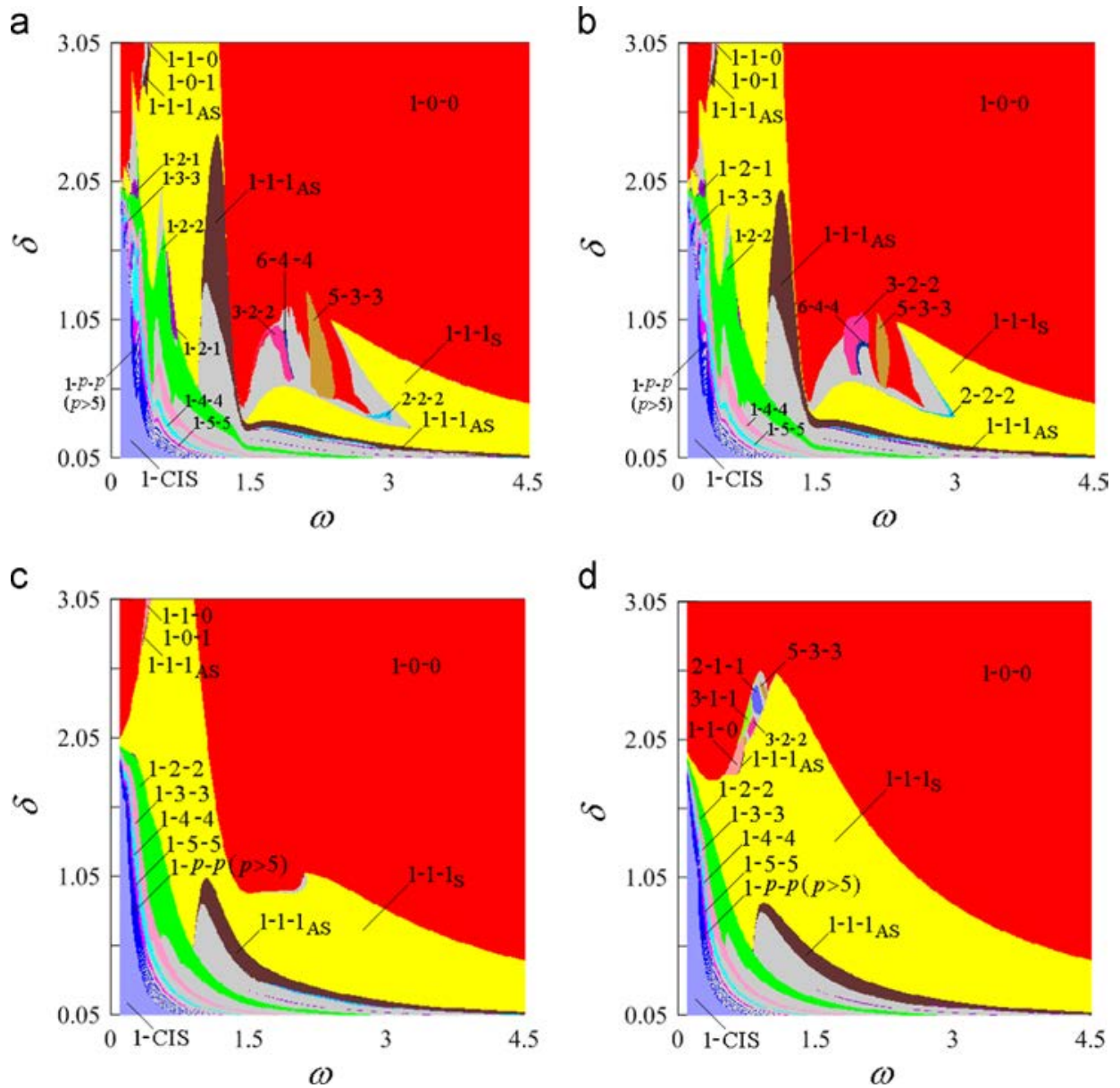

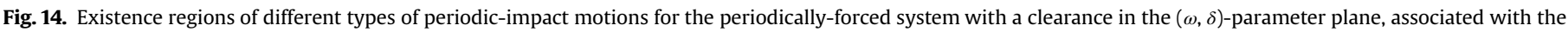
selected values of $\mu_{c}$ and the remainder of the criterion parameters: (a) $\mu_{c}=0.05$; (b) $\mu_{c}=0.25$; (c) $\mu_{c}=0.75$; (d) $\mu_{c}=0.95$.

region of 1-2-2 motion expands gradually with increase in $\mu_{k}$, which is ascertained in comparison to the disturbance region of the motion in Fig. 13(a) and (b). As $\mu_{k}$ is large enough, the stiffness $K_{2}$ connecting the mass block $M_{2}$ and the supporting base is much larger than the stiffness $K_{1}$ connecting two mass blocks so that the amplitude of the mass block $M_{2}$ is much less than that of the mass $M_{1}$. Extremely large $\mu_{k}$ relates to the degenerated case of a singledegree-of-freedom system. For large $\mu_{k}$, the increase of stiffness parameter $\mu_{k}$ brings significant expansion in the existence region of symmetric 1-1-1 motion and slight shrinkage in the existence regions of $1-p-p(p \geq 2)$ and complete chattering-impact motions. The peaks of the existence regions of symmetric 1-1-1 motions of the system with large $\mu_{k}$ are significantly lower in the $(\omega, \delta)$-parameter plane than those with small $\mu_{k}$ so that the tongue-shaped region nearby the critical line $L_{0}: R_{1-0-0} \cap R_{1-1-1}$ between adjacent regions of $1-0-0$ and 1-1-1 motions become larger and more obvious. The phenomenon can be observed by comparing Fig. 2(a), Fig. 13(c) and (d). It is important to note that there exist a series of tongue-shaped regions along left half part of the critical line $L_{0}: R_{1-0-0} \cap R_{1-1-1}$ and their areas become larger and larger with increase in $\delta$. Consequently, the largest tongue-shaped region nearby the critical line $L_{0}: R_{1-0-0} \cap R_{1-1-1}$ always exists near the peak of the existence region of symmetric 1-1-1 motion.

The influence of damping distribution $\mu_{c}$ on dynamics of the system is epitomized in Fig. 14. The correlative relationship between dynamic performance and damping distribution is analyzed to find their reasonable matching law by comparing Figs. 2 and 14. Variation of the parameter $\mu_{c}$ hardly affects the distribution and existence regions of $1-p-p$ motions and complete chattering-impact motion for $\mu_{c}<0.5$, see Fig. 2, Fig. 14(a) and (b). Some subharmonic impact motions, such as 3-2-2 and 5-3-3, etc., are found to exist in small $\mu_{c}$ case. As $\mu_{c}$ is small, the existence region of symmetric 1-1-1 motion is almost divided into two parts, but that of asymmetric 1-1-1 motion shrinks gradually with increasing $\mu_{c}$. For large $\mu_{c}$, the increase of damping parameter $\mu_{c}$ brings large expansion in the existence region of symmetric 1-1-1 motion in small clearance interval. The peak of the existence region of symmetric 1-1-1 motion of the system remarkably reduces with increasing $\mu_{c}$ so that the tongueshaped region nearby the critical line $L_{0}: R_{1-0-0} \cap R_{1-1-1}$ between adjacent regions of 1-0-0 and 1-1-1 motions become more and more obvious, in which $1-1-0$ (or 1-0-1), 2-1-1, 3-1-1, 3-2-2 and 5-3-3 motions exist. The peak of existence region of symmetric 1-11 motion reduces to cause the reduction of regions of other $1-p-p$ motions $(p \geq 2)$, which exist in the zonal zones well arranged, as seen in Fig. 14(c) and (d).

The influence of damping ratio $\zeta$ on dynamics of the system is similar to that of $\mu_{c}$, which is epitomized in Figs. 15 and 16. It is important to note that small damping ratio $\zeta$ can bring about more complex dynamic characteristics than small $\mu_{c}$ does in low exciting frequency range; see Fig. 16(a). The complex distribution of existence regions of $1-p-p$ motions, associated with small $\zeta$, is shown for low frequency interval in Fig. 16. The serious deformation of regions of $1-p-p$ motions generally occurs in enough small $\zeta$ case. As a result, the existence regions of these motions mutually interweave to cause block segmentation which makes the regions of fundamental $1-p-p$ motions $(p \geq 2)$ present irregular distribution characteristics; see Fig. 16(a). For enough small $\zeta$, the regions of long-periodic multi-impact motions unascertained 

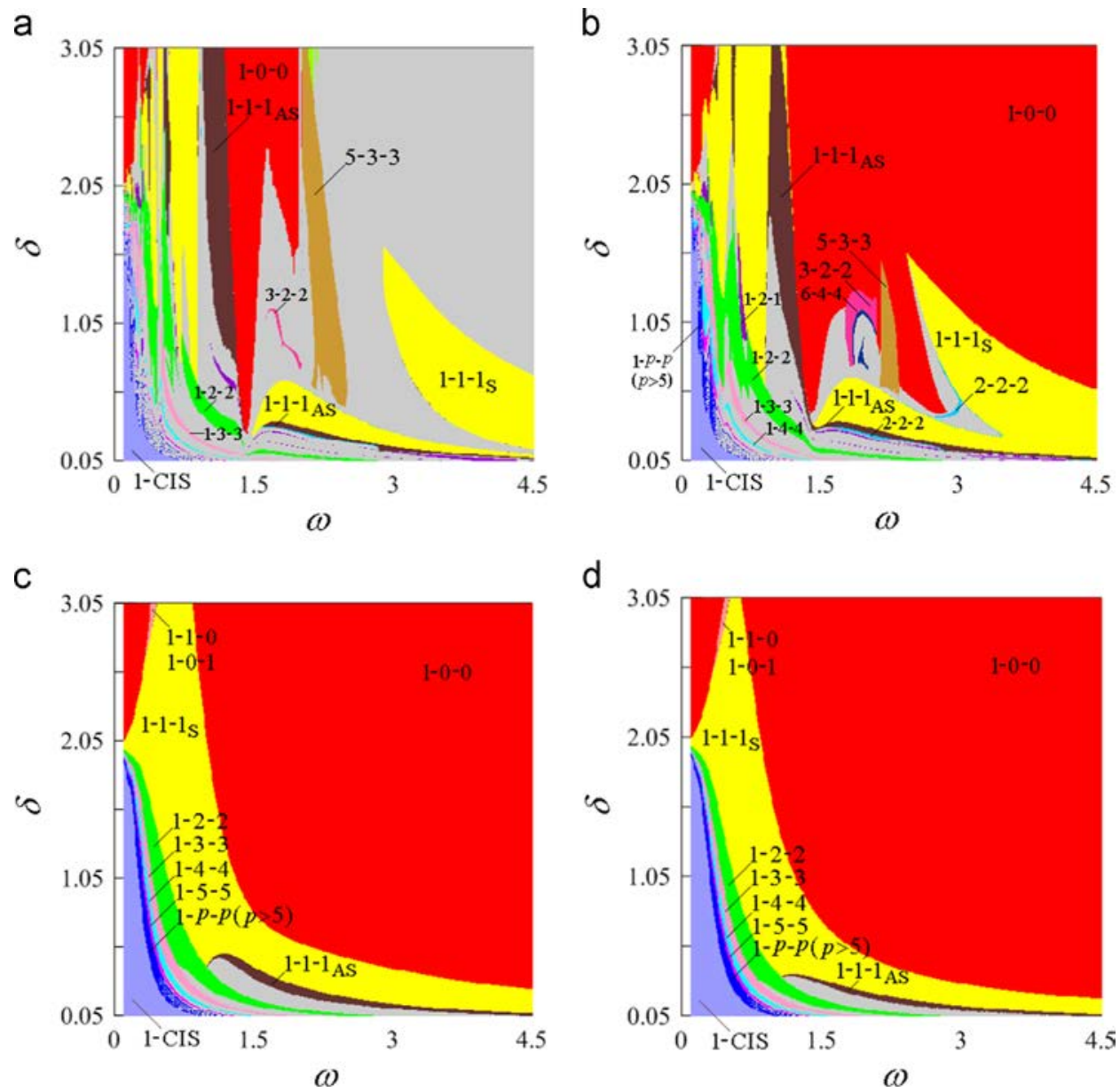

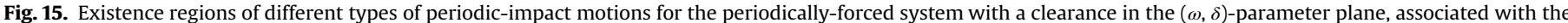
selected values of $\zeta$ and the remainder of the criterion parameters: (a) $\zeta=0.02$; (b) $\zeta=0.05$; (c) $\zeta=0.3$; (d) $\zeta=0.5$.
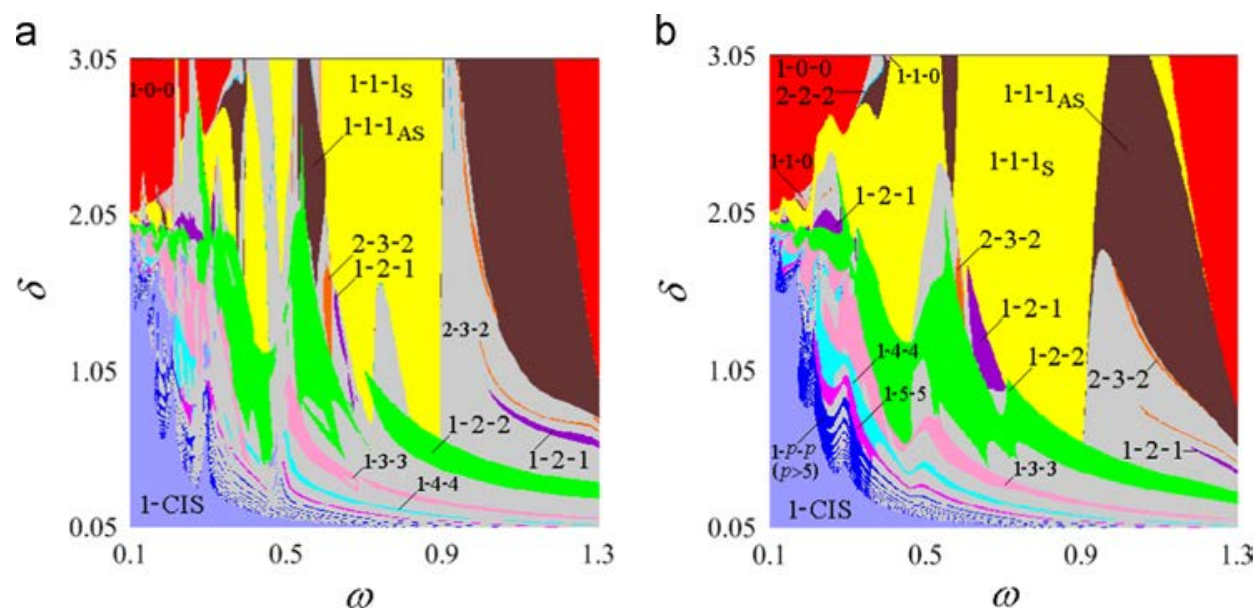

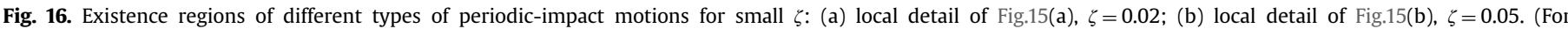
interpretation of the references to color in this figure, the reader is referred to the web version of this article.)

or chaotic motions, marked by blue color, are much larger than that of symmetric 1-1-1 motion in high exciting frequency range; see Fig. 15(a). The reasons that some periodic-impact motions can be not marked are mainly that their existence regions are too small to be observed clearly or the number $n$ of excitation period or the number $p$ of impacts in the whole motion period is too big for the corresponding periodic-impact motions to be ascertained effectively.

Based on the criterion parameters, the correlative relationship between dynamic performance and the exciting forces acting on two mass blocks are studied. The results from simulation, associated with the influence of the exciting forces on main motions of 
a

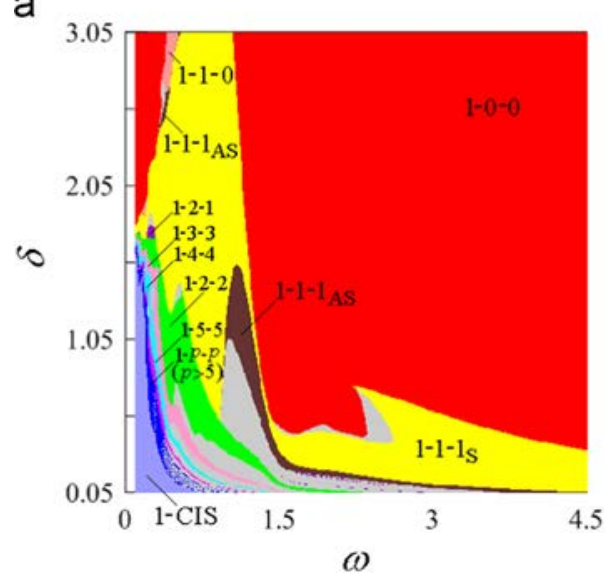

C

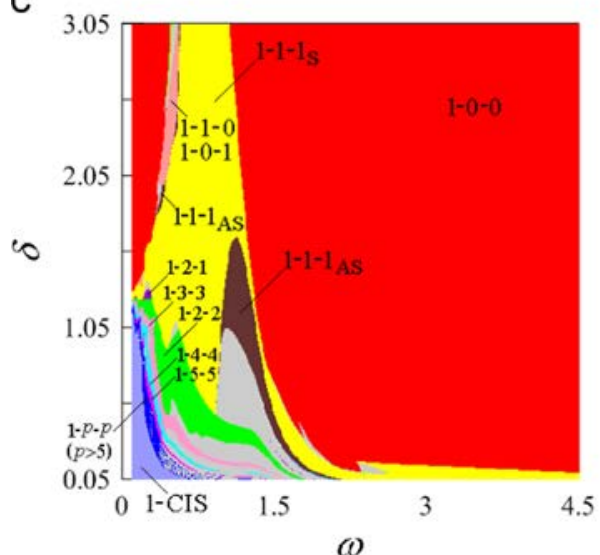

b

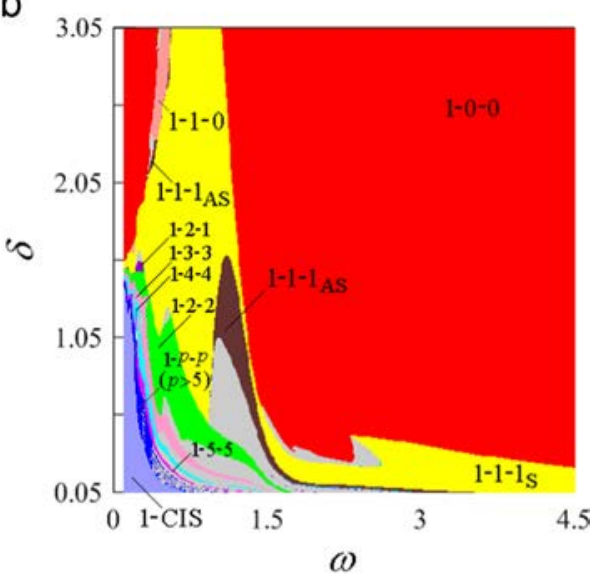

d

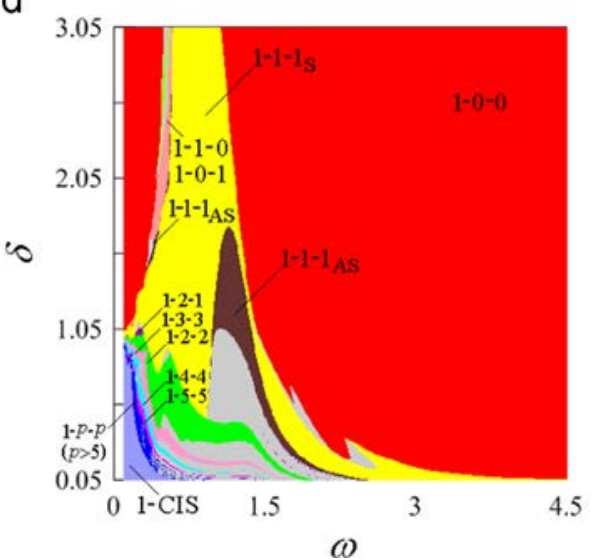

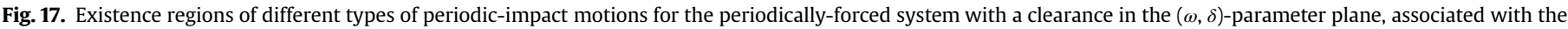
selected values of $f_{20}$ and the remainder of the criterion parameters: (a) $f_{20}=0.25$; (b) $f_{20}=0.5$; (c) $f_{20}=0.75$; (d) $f_{20}=1.0$.

the system, are shown for $f_{20} \in[0,1]$ in Figs. 2, 17 and 25. Variation of the exciting force $f_{20}$ has an effect on the impact velocities and existence regions of $1-p-p$ and complete chattering-impact motions, but it hardly changes general forms of a series of periodic-impact motions shown in Figs. 2 and 17. No anomaly type of periodic-impact motions occurs even for two extreme cases of $f_{20}=0$ and 1 . As the parameter $f_{20}$ is small enough, the exciting force acting on the mass block $M_{1}$ is much larger than that on the other mass, the existence regions of $1-p-p(p \geq 2)$ and complete chattering-impact motions enlarge towards the interval of large clearances, see Fig. 2 and Fig. 17(a). The exciting force acting on the mass block $M_{1}$ gradually decreases whereas that on the other mass correspondingly increases with gradual increase of $f_{20}$. The result causes a gradual reduction of the peak of the existence region of symmetric 1-1-1 motion in the $(\omega, \delta)$-parameter plane so that the existence regions of $1-p-p(p \geq 2)$ and complete chattering-impact motions become correspondingly small. Because the largest tongue-shaped region nearby the critical line $L_{0}: R_{1-0-0} \cap R_{1-1-1}$ always exists near the peak of the existence region of symmetric 1-1-1 motion, a following example is provided to demonstrate that increase of $f_{20}$ leads to a gradual reduction of the peak of the existence region of symmetric 1-1-1 motion. A tongueshaped region nearby the critical line $L_{0}: R_{1-0-0} \cap R_{1-1-1}$ between adjacent regions of $1-0-0$ and 1-1-1 motions becomes larger and more obvious due to a significant reduction of the peak of existence region of symmetric 1-1-1 motion, in which 1-1-0 (or 1-1-0), 3-1$1,2-1-0$ (or 2-0-1),.., etc., fundamental and subharmonic impact motions are found. The positions of these tongue-shaped zones gradually lower and the shape and internal structure of one of them become more and more observable with increase of $f_{20}$, as seen by comparing Figs. 2 and 17. It is interesting to note that change in $f_{20}$ hardly affect the distribution and existence regions of asymmetric 1-1-1 motions in the $(\omega, \delta)$-parameter plane except very small clearance. In high exciting frequency range, the gradual increase of $f_{20}$ leads to smaller and smaller existence region of symmetric 1-1-1 motion.

The influence of restitution coefficient $R$ of impact on dynamics of the system is epitomized in Fig. 18. Based on the existing simulation results for dynamic analysis, the correlative relationship between dynamic performance and the restitution coefficient of impact are analyzed by comparing Figs. 2 and 18. The change in restitution coefficient of impact mainly affects the impact velocities and existence regions of $1-p-p$ and complete chatteringimpact motions. In the interval $R \in[0.05,0.8]$, the total area of existence regions of $1-p-p$ motions $(p \geq 1)$ and complete chattering-impact motions, especially in low exciting frequency range, is basically equal. As the restitution coefficient $R$ of impact is small enough, the existence region of complete chattering-impact motions is very large so that the regions of $1-p-p(p \geq 1)$ motions are badly extruded and substantially reduced. Especially the existence regions of some $1-p-p$ motions $(p \geq 4)$ are too small to be shown in Fig. 18(a) and (b). It is possible that there exist no asymmetric 1-1-1 motion for extreme small $R$. For enough small $R$, the system exhibits subharmonic $2-1-1,3-1-1,3-2-2$ motions and so on in high exciting frequency range; see Fig. 18(a). With increase in $R$, the existence region of complete chattering-impact motion reduces gradually. Consequently, the regions of $1-p-p$ motions correspondingly extends by degrees to the ranges 
a

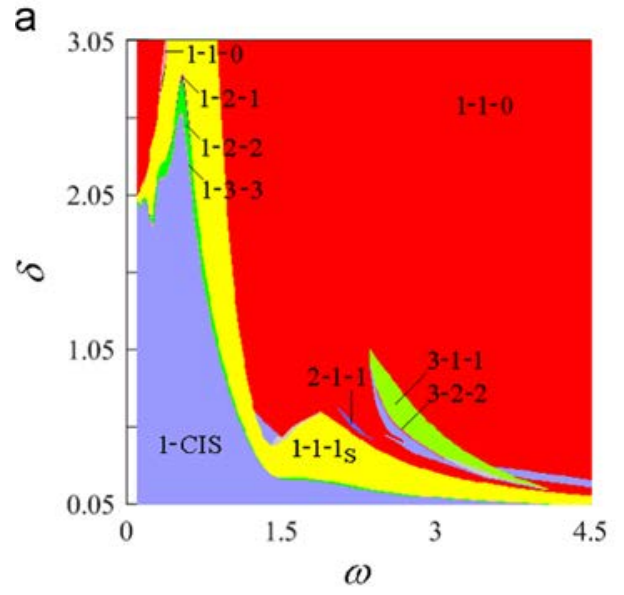

C

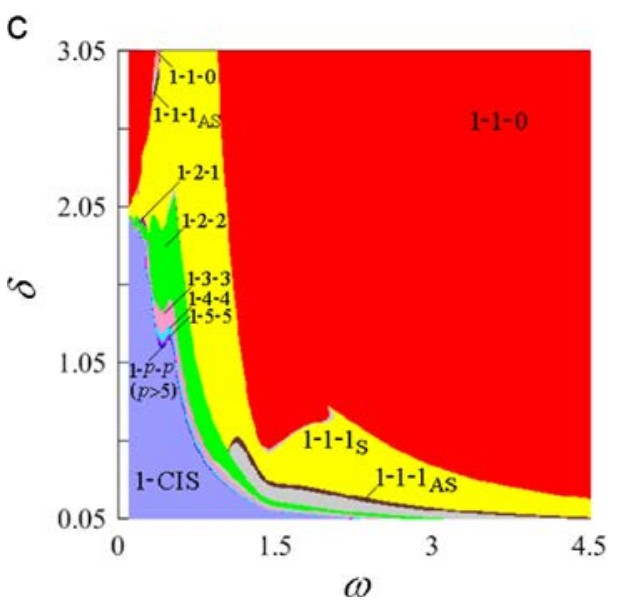

b

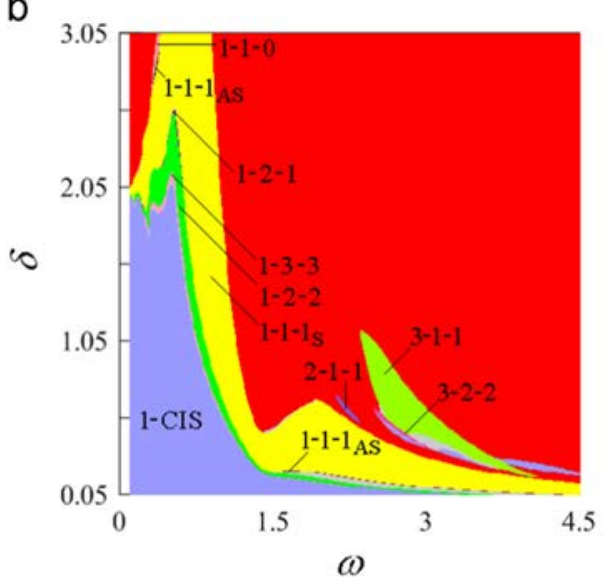

d

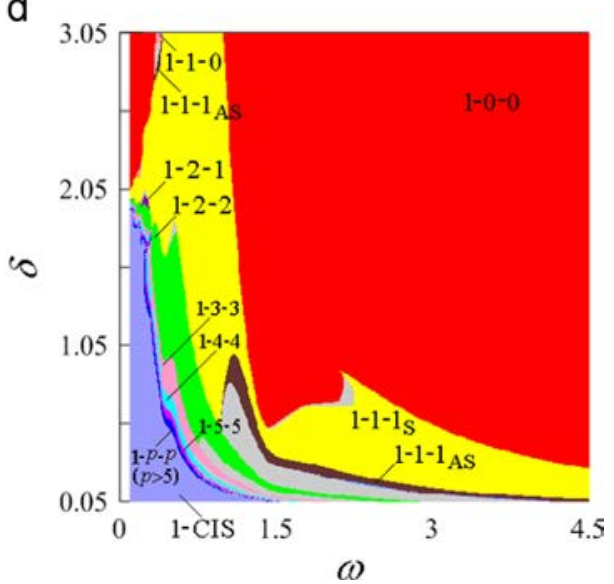

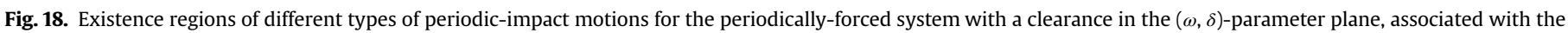
selected values of $R$ and the remainder of the criterion parameters: (a) $R=0.1$; (b) $R=0.2$; (c) $R=0.4$; (d) $R=0.6$.
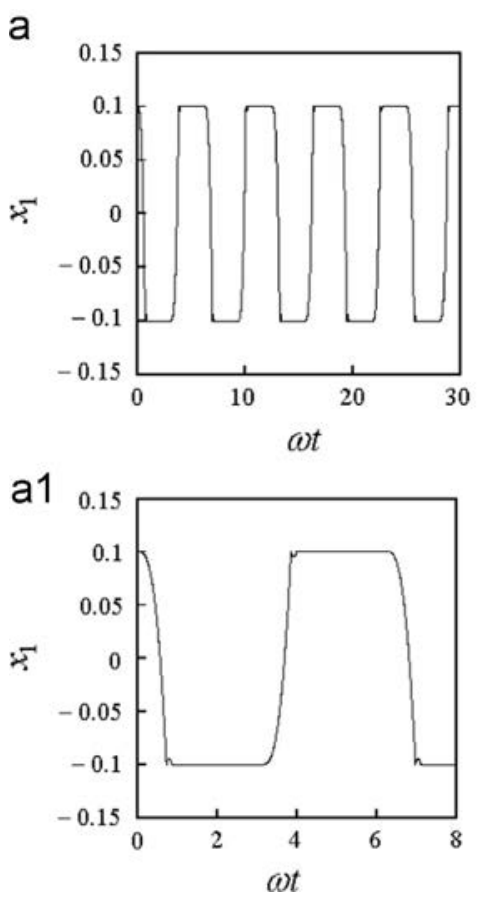

$\mathrm{b}$
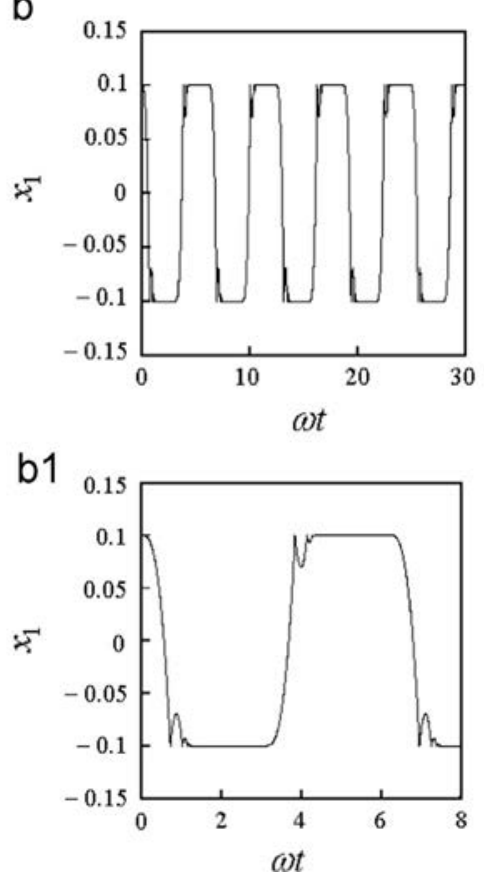

C

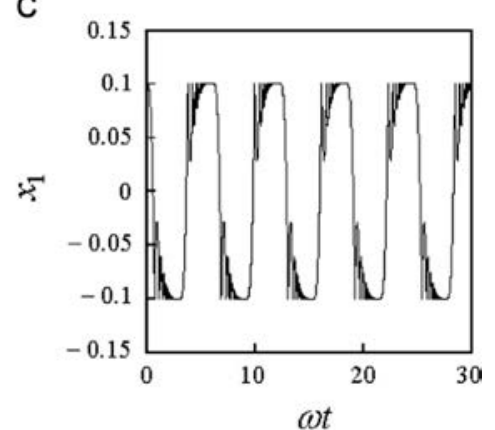

c1

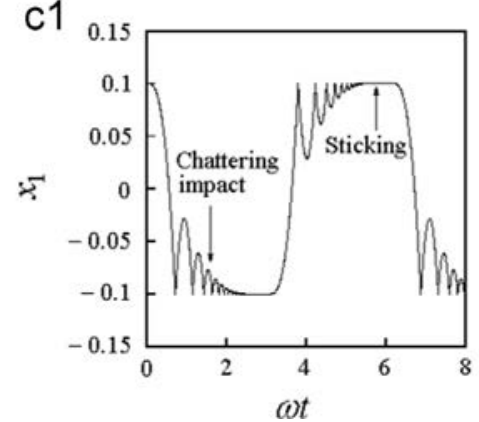

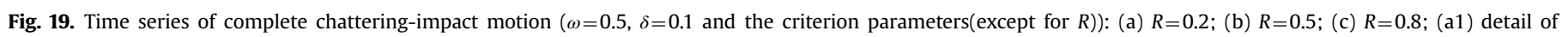
Fig. 19(a); (b1) detail of Fig. 19(b); (c1) detail of Fig.19(c). 

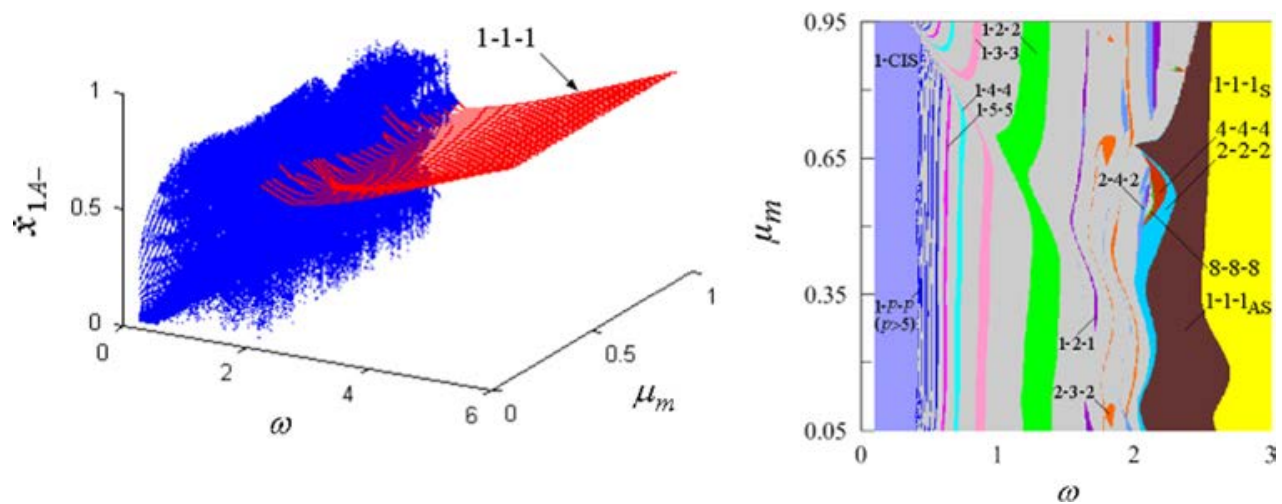

Fig. 20. Before-impact velocities and existence regions of different types of impact motions for the periodically-forced system with a clearance in the $\left(\omega, \mu_{m}\right)$-parameter plane related to $\delta=0.2$ and the remainder of the criterion parameters.
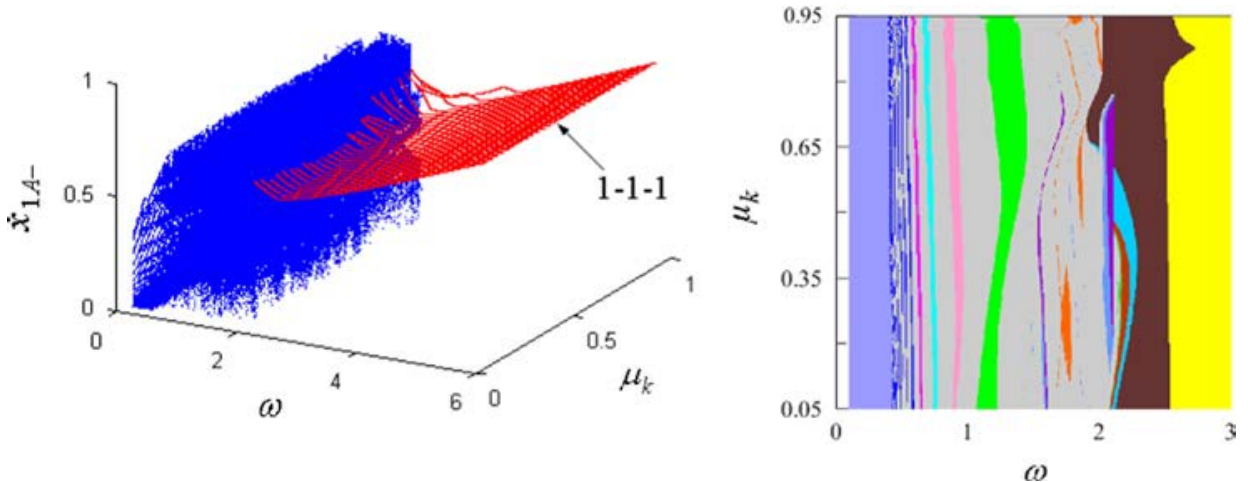

Fig. 21. Before-impact velocities and existence regions of different types of impact motions for the periodically-forced system with a clearance in the $\left(\omega, \mu_{k}\right)$-parameter plane related to $\delta=0.2$ and the remainder of the criterion parameters.
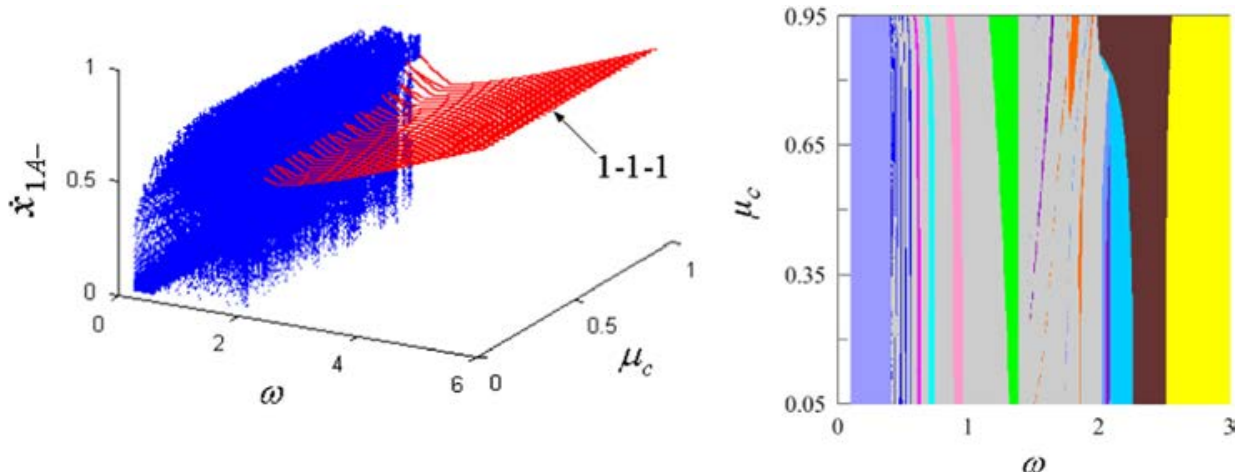

Fig. 22. Before-impact velocities and existence regions of different types of impact motions for the periodically-forced system with a clearance in the $\left(\omega, \mu_{c}\right)$-parameter plane related to $\delta=0.2$ and the remainder of the criterion parameters.
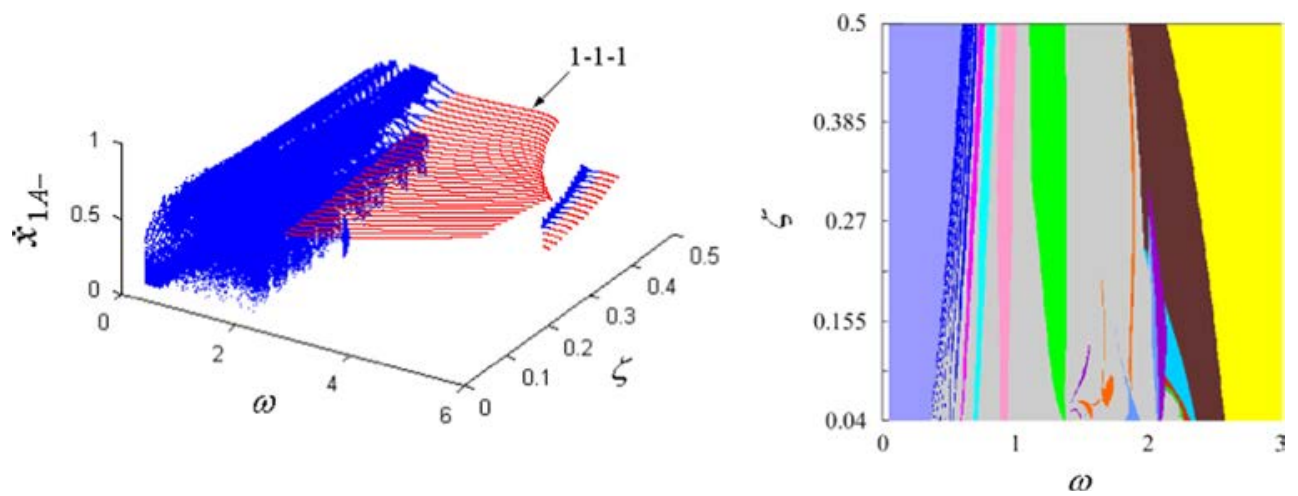

Fig. 23. Before-impact velocities and existence regions of different types of impact motions for the periodically-forced system with a clearance in the ( $\omega$, $\zeta$ )-parameter plane related to $\delta=0.2$ and the remainder of the criterion parameters. 

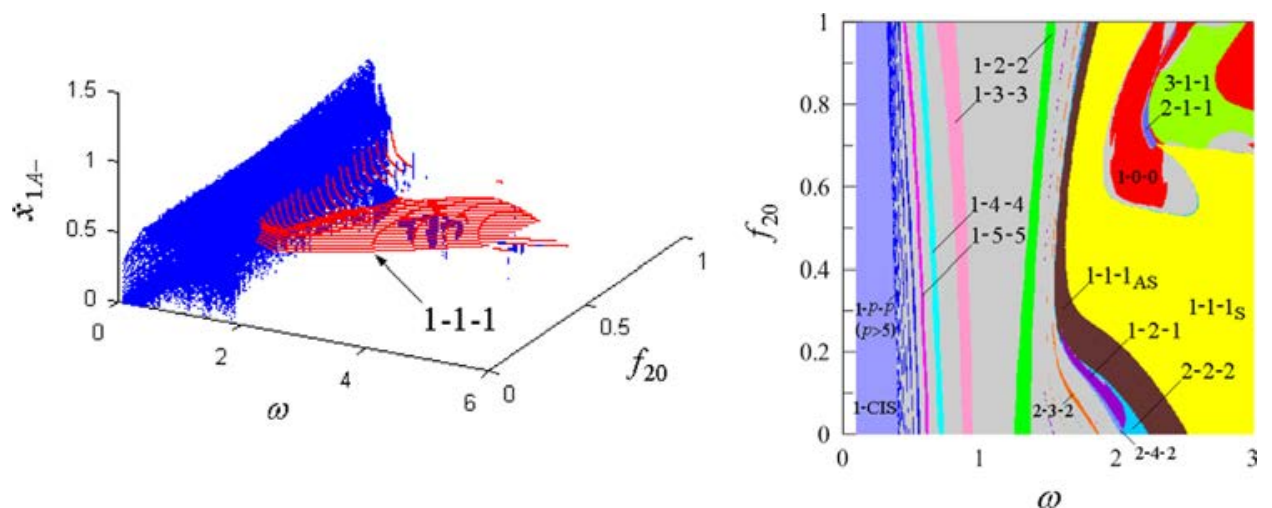

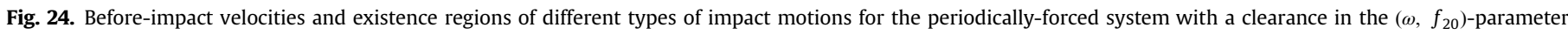
plane related to $\delta=0.2$ and the remainder of the criterion parameters.
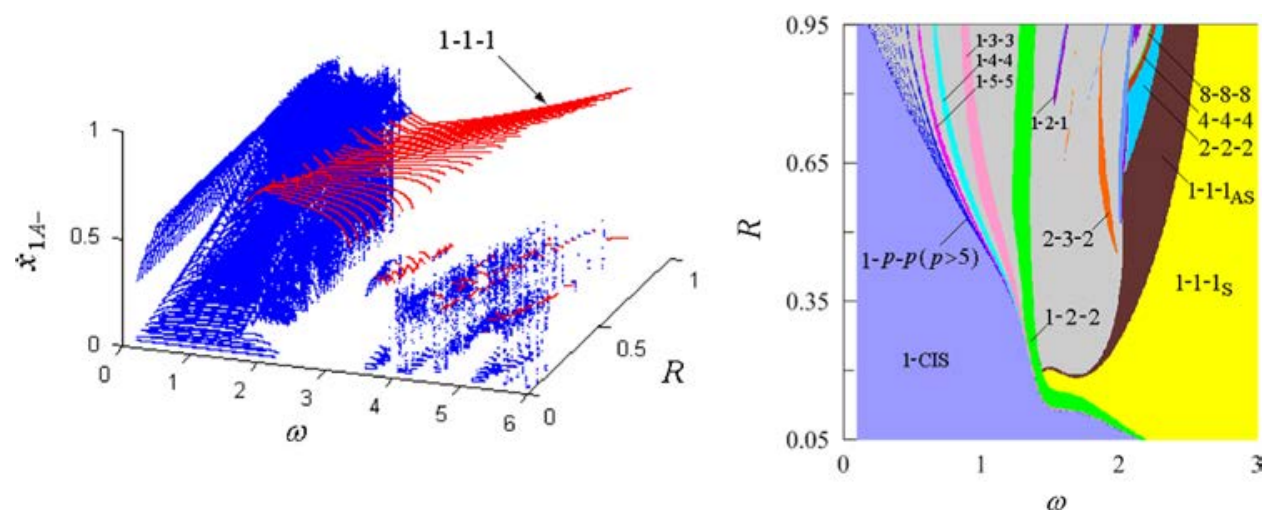

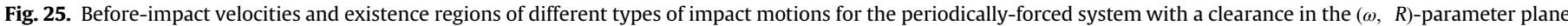
related to $\delta=0.2$ and the remainder of the criterion parameters.
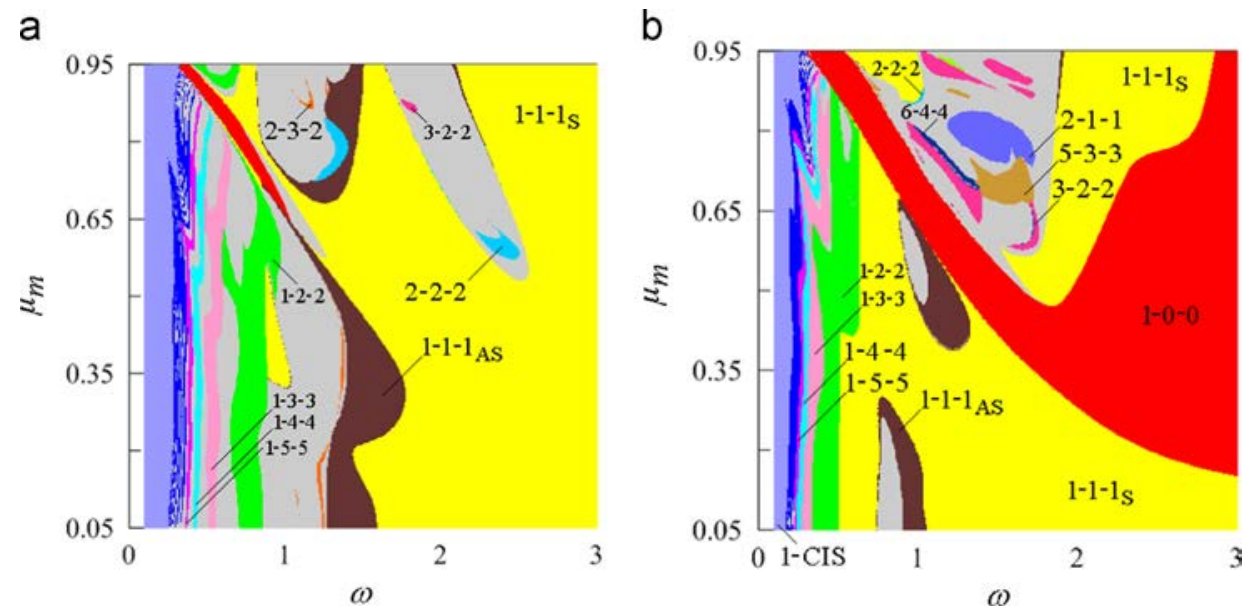

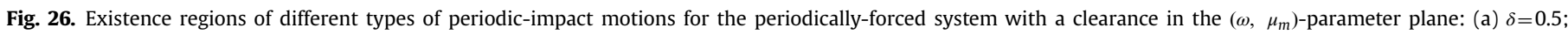
(b) $\delta=1.0$.

observed obviously. Correspondingly, the region of symmetric 1-1-1 motion becomes wider in the exciting frequency interval and the existence region of asymmetric 1-1-1 motion extends slightly. On the other hand, the restitution coefficient of impact brings also great influences on the impact sequences and the sticking times of $1-\bar{p}-\bar{p}$ motion in a motion period. For large restitution coefficient $R$, the impact sequence of $1-\bar{p}-\bar{p}$ motion is also more obvious in the motion period and the sticking time correspondingly shorter. Conversely, as $R$ is small enough, the impact strength is markedly attenuated, the impact sequence of $1-\bar{p}-\bar{p}$ motion decays quickly in the motion period and becomes not obvious in visual sensation and quantity (but the chatter sequence is still infinite), the sticking time is correspondingly longer. A simulation result, associated with $\omega=0.5, \delta=0.1$ and the remainder of the criterion parameters, is provided to observe the influence of restitution coefficient $R$ on the impact number and the sticking time of $1-\bar{p}-\bar{p}$ motion in a motion period, which is shown for $R=0.2,0.5$ and 0.8 in Fig. 19 . 
a

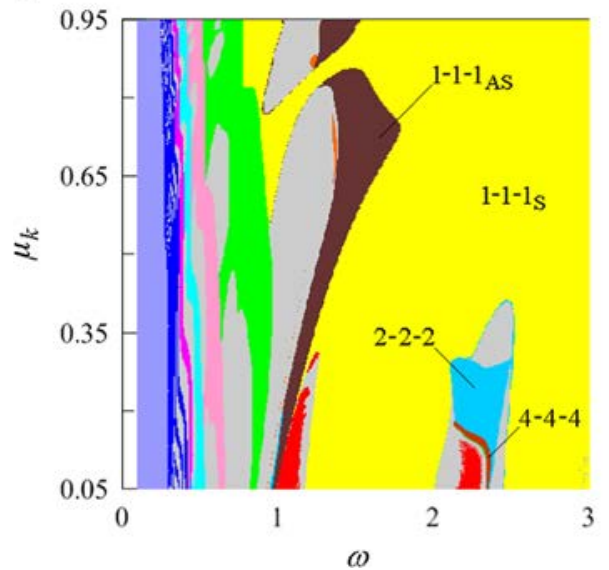

b

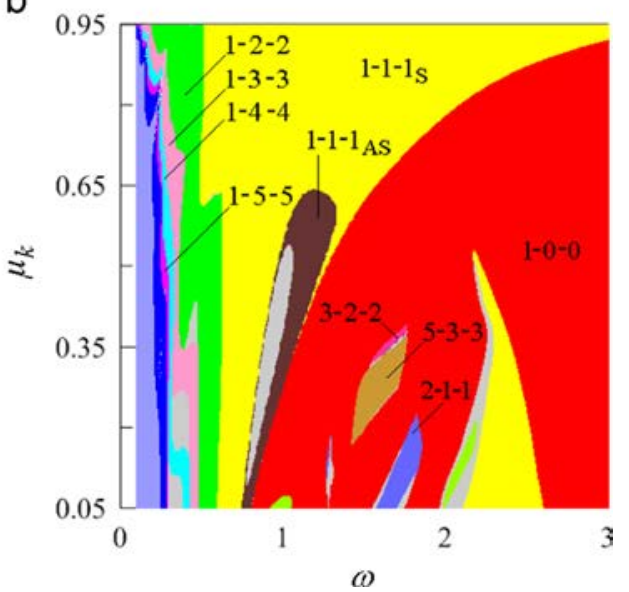

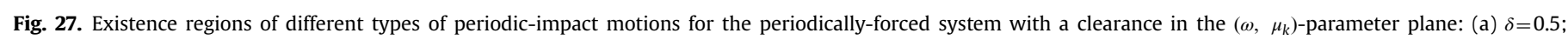
(b) $\delta=1.0$.

a

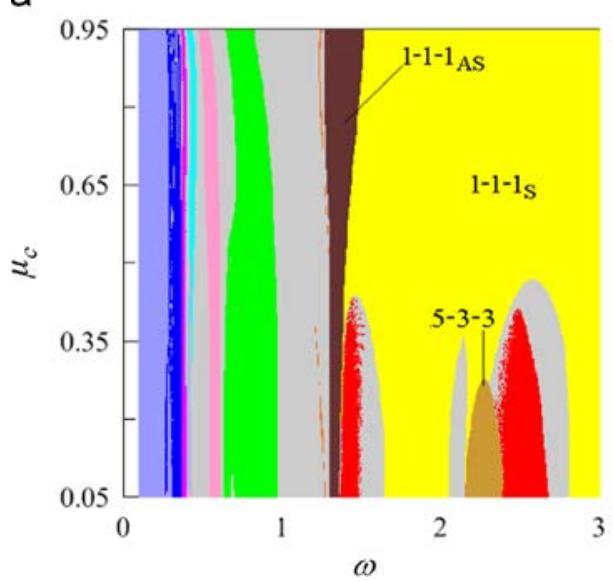

b

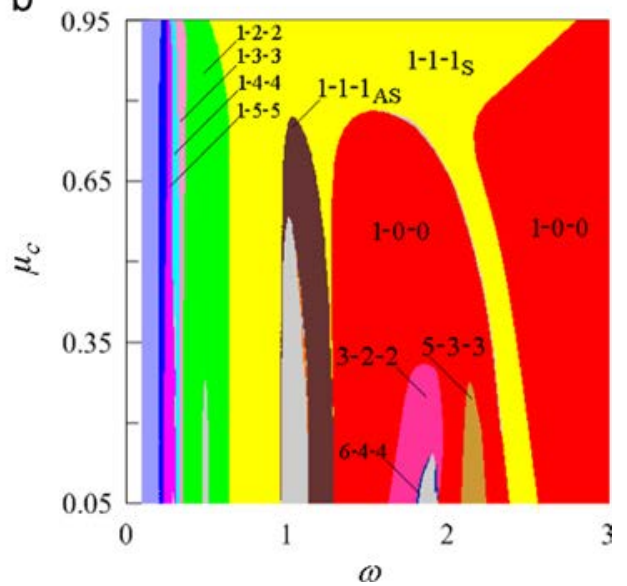

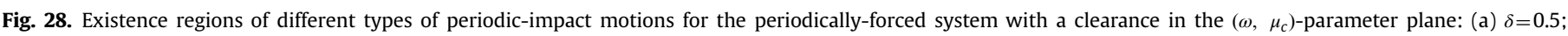
(b) $\delta=1.0$.

\section{The influence of system parameters to before-impact velocity}

The clearances or constraints among mechanical components exist inevitably in mechanical systems due to the restriction on technological level in the mechanical machining process, the demand for mechanical design and assembly errors, the need for the heat-expansion and cold-contraction of some part of mechanical components or other reason. The existence of the clearances or constraints is bound to bring about the vibro-impact effects in service of the mechanisms. The vibroimpact effects have a great influence on performance of mechanical equipments, increase noise levels and intensify fatigue and abrasion of mechanical parts. An important scientific research subject in the field of mechanical engineering is urgent to be solved, i.e., how to reduce wear of mechanical components and improve use efficiency, service life and operation reliability of non-smooth mechanical systems. Therefore, it is a primary purpose of dynamic research on mechanical vibrating systems with clearances or constraints how both vibration and impact are effectively utilized or furthest decreased to prevent from chattering-impact or chaotic impact motions so as to restrain noise levels and reduce the abrasion between components and constraints.

The influence of clearance nonlinearity on before-import velocity of the mass block $M_{1}$ is epitomized in Fig. 2(b). The diagram is useful when the distribution of impact velocity is of interest over the whole $(\omega, \delta)$ plane. Based on the simulation results of the system with the criterion parameters, the correlative relationship between before-impact velocity and parameters $(\omega, \delta)$ is analyzed to find their reasonable matching law. Impact velocities of symmetric and asymmetric 1-1-1 motions are specially marked in Fig. 2(b) by red color, the existence regions of which are shown in Fig. 2(a). With increase in the value of clearance, the impact velocity of 1-1-1 motion, associated with small $\delta$, raises gradually. The resonance peak of function curve $\dot{x}_{1 A-}(\omega)$ of impact velocity of symmetric 1-1-1 motion, corresponding to large $\delta$, rises gradually with increasing $\delta$. The regularities can be observed by Fig. 2(b). In general case, small clearances lead to low impact velocity of symmetric 1-1-1 motion. The function curve $\dot{x}_{1 A_{-}}(\omega)$ of impact velocity of symmetric 1-1-1 motion, associated with $\delta=0.2$, is lower on the whole than that corresponding to $\delta>0.2$. As the clearance is located at the interval of approximately [0.6, $2.0], 1-p-p$ motions $(p \geq 2)$, complete chattering-impact motions, $1-(p+1)-p, 2-(2 p+1)-2 p$ and $3-(3 p+1)-(3 p+1)$ motions $(p \geq 1)$ in tongue-shaped zones play major roles in low exciting frequency range, respectively. Consequently, these motions bring about the increase of average impact number in an exciting period, which increases noise levels and intensifies wear of the impact mass and stops. The choice of small clearance and the determination of reasonable parameter matching and effective exciting 
a

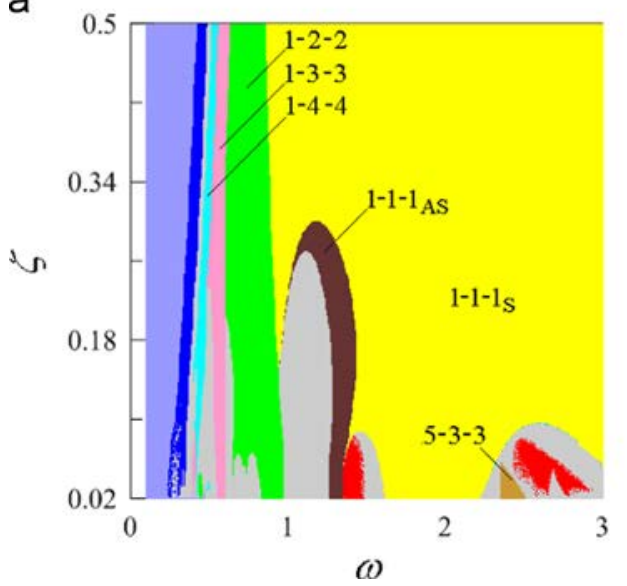

b

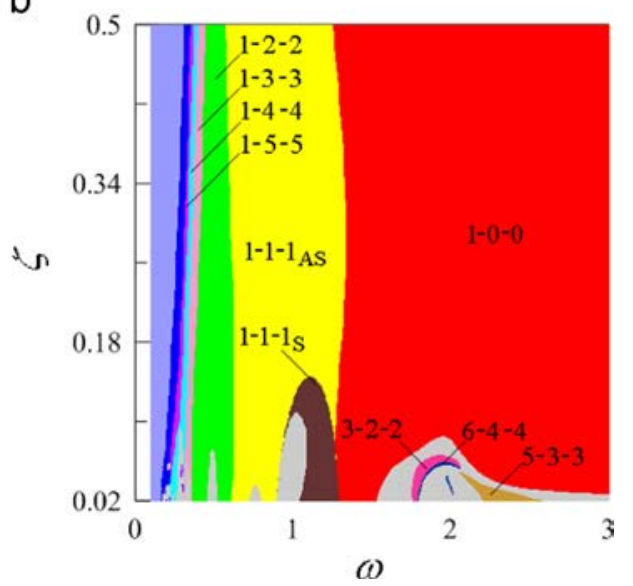

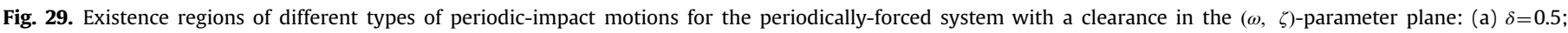
(b) $\delta=1.0$.

a

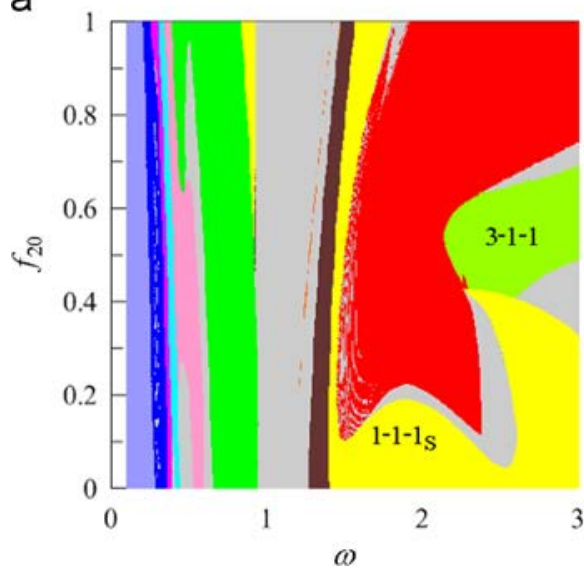

b

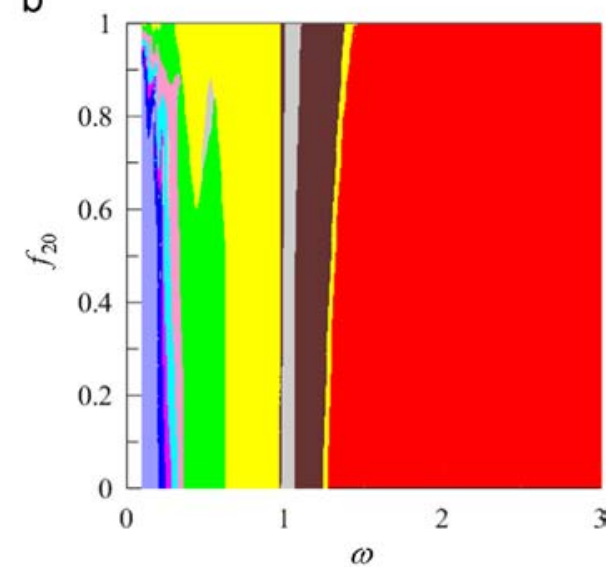

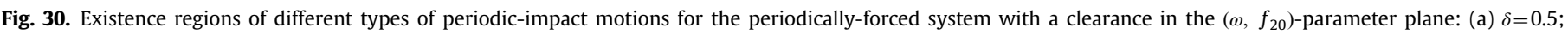
(b) $\delta=1.0$.

frequency range are conducive to obtain symmetric 1-1-1 motion, reduce its impact strength, abrasion and noise levels.

A conclusion has been obtained from Fig. 2 that the function curve $\dot{x}_{1 A-}(\omega)$ of before-impact velocity of $1-1-1$ motion, associated with the criterion parameters and $\delta=0.2$, is lower on the whole than those corresponding to $\delta>0.2$. Based on the conclusion above-mentioned, the influence of parameter variation on before-impact velocity of the mass block $M_{1}$ is epitomized in Figs. 20-25. The correlative relationship between impact velocity $\dot{x}_{1 A-}$ and parameters $\left(\omega, \mu_{m}\right)$ are shown for $\delta=0.2$ in Fig. 20. The change in $\mu_{m}$ hardly affects the impact velocity of the mass block $M_{1}$ for the same exciting frequency, and the range of exciting frequency of symmetric 1-1-1 motion is basically constant for small clearance and large mass distribution. As for the same mass distribution, impact velocity of symmetric 1-1-1 motion gradually raises with increase in $\omega$. As for the mass distribution interval $\mu_{m} \in[0.05,0.95], 1-p-p$ fundamental motions $(p \geq 2)$, complete chattering-impact motions, $1-2-1,2-3-2$ and $2-4-2$ motions and chaos, etc., dominate in the exciting frequency range of $\omega<2$, respectively. These motions bring about the increase of average impact number in a motion period. Whether large or small $\mu_{m}$, these motions have the negative effects on the vibrating system associated with small clearances and low exciting frequencies. With increase in $\delta$, large $\mu_{m}$ causes that the existence region of symmetric 1-1-1 motion becomes relatively small in the exciting frequency range. Two examples associated with $\delta=0.5$ and 1 are provided for analyzing the influence of parameters $\delta$ and $\mu_{m}$ on dynamic performance of the system. As for a little larger clearance, the existence region of symmetric 1-1-1 motion in the $\left(\omega, \mu_{m}\right)$-parameter plane is divided into two parts by that of 1-0-0 motion, and subharmonic 2-1-1, 3-2-2, 5-3-3, 6-4-4 and chaotic motions, etc., are found to appear in the interval of large $\mu_{m}$; see Fig. 26.

The correlative relationship between before-impact velocity $\dot{x}_{1 A-}$ and parameters $\left(\omega, \mu_{k}\right)$ are shown for $\delta=0.2$ in Fig. 21 . It is interesting to note that the change in $\mu_{k}$ hardly affects the exciting frequency range of existence regions of $1-p-p$ fundamental motions $(p>3)$ and complete chattering-impact motions associated with small $\delta$. The existence region of symmetric 1-1-1 motion is almost invariant in a certain range of exciting frequency as $\mu_{k}$ is not large enough, and the impact velocity of the motion is basically constant whether $\mu_{k}$ increases or decreases for the same exciting frequency. The range of exciting frequency of asymmetric $1-1-1$ motion slightly increases as $\mu_{k}$ is large enough. As for a little larger clearance $\delta$, small $\mu_{k}$ causes that the existence region of symmetric 1-1-1 motion becomes narrow in the exciting frequency range, and subharmonic 2-1-1 motion is found to exist in the range of $\mu_{k} \in(0.05,0.2377)$; the existence region of symmetric 1-1-1 motion, associated with large $\mu_{k}$, is still wide in the exciting frequency range, as seen in Fig. 27. Dynamic performance of the 
a

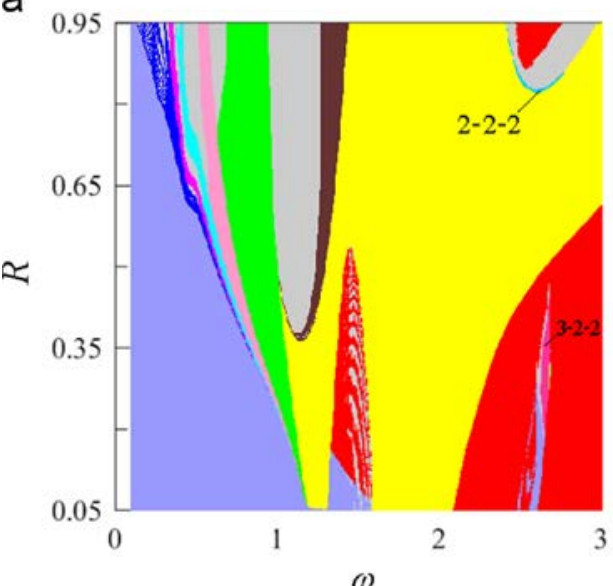

b

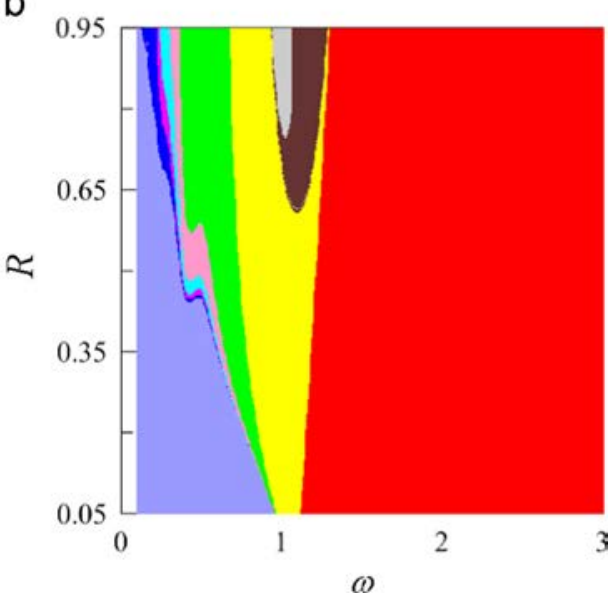

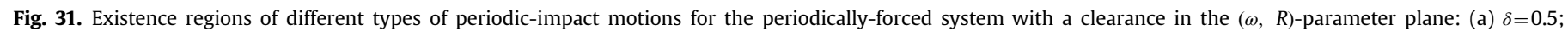
(b) $\delta=1.0$.

system can be improved significantly by selecting slightly larger stiffness distribution $\mu_{k}$.

The correlative relationship between before-impact velocity $\dot{x}_{1 A}$ - and parameters $\left(\omega, \mu_{c}\right)$ are shown for $\delta=0.2$ in Fig. 22 . As the damping distribution $\mu_{c}$ is not large enough, the existence regions of $1-p-p$ motions $(p \geq 3)$ and complete chattering-impact motions are almost invariant in $\omega$ interval. The range of exciting frequency of symmetric 1-1-1 motion is almost invariant for $\mu_{c}$, and impact velocity of the motion is basically constant for the same exciting frequency with change in $\mu_{c}$. The range of exciting frequency of asymmetric 1-1-1 motion slightly increases as $\mu_{c}$ is large enough. As for small clearance, small damping distribution $\mu_{c}$ hardly affects dynamics of the system, as seen in Fig. 22. As for a little larger clearance $\delta$, small $\mu_{c}$ causes that the existence region of symmetric 11-1 motion becomes narrow in the exciting frequency range and the system exhibits subharmonic 3-2-2 and 5-3-3 motions in a little higher exciting frequency interval; see Fig. 28.

The correlative relationship between impact velocity $\dot{x}_{1 A-}$ and parameters $(\omega, \zeta)$ is shown for $\delta=0.2$ in Fig. 23. With increase in $\zeta$, impact velocity of 1-1-1 motion, corresponding to the same and relatively small exciting frequency, slightly attenuates at the outset to the lower bound and after that begins to slightly increase. As the exciting frequency keeps invariant and is higher than some value, impact velocity of 1-1-1 motion attenuates gradually with increasing $\zeta$. It is important to note that the function curve $\dot{x}_{1 A-}(\omega)$ of symmetric 1-1-1 motion is divided into two sections in the parameter range associated with large $\zeta$ and higher $\omega$. The section of the function curve $\dot{x}_{1 A-}(\omega)$ of symmetric 1-1-1 motion, in low exciting frequency range, is higher than the other in high exciting frequency interval, both are mainly separated by the windows of quasi-periodic, chaotic and impactless motions. There exists Neimark-Sacker bifurcation of symmetric 1-1-1 motion as $\zeta$ is very small or large enough. As for large $\zeta$, impact velocity of symmetric 1-1-1 motion is very small in certain a range of high exciting frequency; see Fig. 23. The correlative relationship between dynamic performance and parameters $(\omega, \zeta)$ are shown for $\delta=0.5$ and 1 in Fig. 29. Small $\zeta$ badly affects dynamics of the system, slightly large $\zeta$ leads to more energy consumption of the system to reduce its use efficiency. Based on comprehensive consideration of large existence region and low impact velocity of 1-1-1 motion, the damping ratio range $\zeta \in[0.1,0.2]$ is feasible and effective to the operation reliability of the system.

The correlative relationship between before-impact velocity $\dot{x}_{1 A}$ - and parameters $\left(\omega, f_{20}\right)$ are shown for $\delta=0.2$ in Fig. 24. The change in $f_{20}$ mainly affects the existence regions of 1-1-1 motions associated with small $\delta$ in high exciting frequency range. As $f_{20}$ is large enough, the existence region of $1-1-1$ motion is very narrow for small $\delta$ in the $\left(\omega, f_{20}\right)$-parameter plane, as seen in Figs. 17 and 24 and Fig. 30(a). With increase in parameter $f_{20}$, impact velocity of symmetric 1-1-1 motion, corresponding to the same exciting frequency, attenuates gradually. As for invariant exciting frequency, the impact strength of complete chatteringimpact motions can enhance gradually with increasing $f_{20}$. As for small clearances, large $f_{20}$ causes that the existence region of symmetric 1-1-1 motion becomes narrower in the exciting frequency range; see Fig. 17 and Fig. 30(a).

The correlative relationship between before-impact velocity $\dot{x}_{1 A-}$ and parameters $(\omega, R)$ is shown for $\delta=0.2$ in Fig. 25. As the restitution coefficient $R$ of impact is not large enough, the system exhibits complete chattering-impact motions $(1-\bar{p}-\bar{p})$ in a large exciting frequency interval due to fast attenuation in impact velocities, though the first impact velocities of these motions in an exciting period are very high. Furthermore, the existence regions of $1-p-p$ motions $(p \geq 3)$, associated with small $R$, are too narrow in $\omega$ interval to be observed. It is important to note that the highest branches of function curves $\dot{x}_{1 A-}(\omega)$ of $1-\bar{p}-\bar{p}$ motions, in certain an interval of $\omega$, are possibly higher than the whole curve $\dot{x}_{1 A-}(\omega)$ of 1-1-1 motion for small $R$. With increase in the restitution coefficient $R$, the existence region of complete chattering-impact motions is gradually diminished in the $\omega$ interval and those of $1-p-p$ motions accordingly expand, and impact velocity of 1-1-1 motion, corresponding to the same and relatively small exciting frequency, slightly increases at the outset to the upper bound and after that begins to slightly attenuate. However, as the exciting frequency is higher than some value and keeps invariant, impact velocity of 1-1-1 motion increases gradually with increasing $R$. As for small $R$, the function curves $\dot{x}_{1 A-}(\omega)$ of impact velocity of symmetric $1-1-1$ motion attenuate gradually with increasing the exciting frequency $\omega$. The case is contrary after $R$ is larger than some value, the function curves $\dot{x}_{1 A-}(\omega)$ of symmetric 1-1-1 motion increases progressively with increasing $\omega$. As $R$ is not large enough, some narrow windows of 1-1-1 motions exist in high exciting frequency range, which are isolated by other periodic-impact or chaotic motions. As for a little larger clearance $\delta$, small $R$ leads to narrower existence region of symmetric 1-1-1 motion in the exciting frequency range; see Fig. 31. Some characteristics of complete chattering-impact motions must be emphasized. As $R$ is small enough, the impact strength is markedly attenuated, the impact sequence of $1-\bar{p}-\bar{p}$ motion decays quickly in the motion period and becomes not obvious in visual sensation and 
quantity (but the chatter sequence is still infinite), and the sticking time is correspondingly longer. Conversely, for large restitution coefficient $R$, the impact sequence of $1-\bar{p}-\bar{p}$ motion is also more obvious in the motion period and the sticking time becomes correspondingly shorter due to slow attenuation in impacts. The effect, i.e., large impact velocities and increase of average impact number in a motion period, badly intensifies abrasion and pitting fatigue between the restrained mass and clearances and increase noise levels. It is valuable to avoid the existence regions of complete and incomplete chattering-impact motions and $1-p-p$ motions $(p \geq 2)$ in parameter design as much as possible to improve service life and operation reliability of vibrating systems with clearances or constraints.

\section{Conclusions}

In this paper we consider a two-degree-of-freedom periodicallyforced system with a clearance represented by two symmetric rigid stops. The correlative relationship between dynamic performance and system parameters is analyzed to find their reasonable matching law. Two key parameters of the system, the dimensionless excitation frequency $\omega$ and clearance $\delta$, are emphasized to analyze the influence of the main factors on dynamics of the system.

The system usually exhibits $1-p-p$ motions $(p \geq 1)$ and complete chattering-impact motions in low exciting frequency or small clearance range. As the clearance $\delta$ is small or small enough, the transition between adjacent fundament motions basically goes through three processes with decrease in $\omega$, which are epitomized as follows: pitchfork bifurcation of symmetric $1-p-p$ motion; period-doubling bifurcation of asymmetric $1-p-p$ motion; nonperiodic or chaotic motions caused by a succession of perioddoubling bifurcations; symmetric $1-(p+1)-(p+1)$ motion generated by a degeneration of chaos. Both $1-p-p$ and $1-(p+1)-(p+1)$ motions are separated by a region including other periodic-impact and chaotic motions, in which only the zones of $3-(p+1)-(p+1)$ and $1-(p+1)-p$ motions are obvious enough to be observed.

For slightly large clearance $\delta$, the transition from $1-p-p$ to $1-(p+1)-(p+1)$ motion arises with decreasing the exciting frequency $\omega$ up to the grazing bifurcation boundary $\mathrm{G}_{1-p-p}$ of $1-p-p$ motion. A series of grazing bifurcations occur with decreasing $\omega$ so that the number $p$ of impacts of the fundamental group of motions increases two by two. As $p$ becomes big enough, $1-p-p$ motion will exhibit incomplete chattering-impact characteristics, which is the so-called an incomplete chattering sequence in an excitation period followed by a finite sequence of impacts with successively reduced velocity. Complete chattering-impact motion with sticking will occur with decreasing $\omega$ up to the sliding bifurcation boundary. Transition from $1-(p+1)-(p+1)$ to $1-p-p$ motion occurs with increasing $\omega$ up to the saddle-node bifurcation boundary $\mathrm{SN}_{1-(p+1)-(p+1)}$ of $1-$ $(p+1)-(p+1)$ motion. The transition between neighbor fundamental motions $1-p-p$ and $1-(p+1)-(p+1)$ is generally non-reversible. There exist a series of singular points on the boundaries between the existence regions of $1-p-p$ and $1-(p+1)-(p+1)$ motions, at which different saddle-node bifurcation boundaries of $1-(p+1)-(p+1)$ motion, real-grazing and bare-grazing boundaries of $1-p-p$ motion alternately and mutually intersect. Consequently, two types of transition regions (hysteresis and tongue-shaped regions) are created, by which neighboring fundamental impact motions are separated. The hysteresis regions are limited at the upper bound by the saddle-node bifurcation boundaries $\mathrm{SN}_{1-(p+1)-(p+1)}^{1-p-p}$ of $1-(p+1)-$ $(p+1)$ motion and at the lower bound by the real-grazing boundaries $\mathrm{G}_{1-p-p}$ of $1-p-p$ motion. The tongue-shaped regions are limited at the upper bound by the bare-grazing boundary $G_{1-p-p}^{b}$ of $1-p-p$ motion and at the lower bound by the saddle-node bifurcation boundary $\mathrm{SN}_{1-(p+1)-(p+1)}^{1-(p+1)-p}$ of $1-(p+1)-(p+1)$ motion. A series of zones of fundamental $1-(p+1)-p$, subharmonic $3-(3 p+1)-(3 p+1)$ and $2-(2 p+1)-2 p$ impact orbits $(p \geq 0)$ dominate in the tongueshaped regions between adjacent regions of $1-p-p$ and $1-(p+1)-$ $(p+1)$ motions.

As for large clearance $\delta$, the system exhibits mainly 1-0-0 or symmetric 1-1-1 motion in most regions of the $(\omega, \delta)$-parameter plane except small or narrow tongue-shaped zones nearby the critical line $L_{0}: R_{1-0-0} \cap R_{1-1-1}$, in which there exist narrower zones of fundamental 1-1-0 (or 1-0-1), subharmonic 3-1-1 and 2-1-0 (or 2-0-1) motions.

The system exhibits more complex dynamical characteristics as $\mu_{m}$ becomes large enough. Consequently, more types of periodicimpact motions appear and the existence region of complete chattering-impact motions is extended to larger clearance interval. It is possible for more complex sequences of subharmonic impact motions to occur in large $\mu_{m}$ case, such as $2-1-1,3-1-1,3-2-2$ and $5-3-3$, etc. As for small $\mu_{k}$, the existence regions of $1-p-p$ motions $(p \geq 2)$ are extended to larger clearance interval and the existence regions of symmetric 1-1-1 motion are divided into two parts. Many complex sequences of subharmonic impact motions are found to exist in small $\mu_{k}$ case. The increase of stiffness parameter $\mu_{k}$ brings significant expansion in the existence region of symmetric 1-1-1 motion and obvious shrinkage in the existence regions of $1-p-p(p \geq 2)$ and complete chattering-impact motions. As the damping distribution $\mu_{c}$ is not large enough, its variation hardly affects the distribution and existence regions of $1-p-p$ and complete chattering-impact motions. As for large $\mu_{c}$, the exciting frequency range of symmetric 1-1-1 motion, associated with small clearance interval, becomes correspondingly large. The serious deformation of regions of $1-p-p$ motions generally occurs in enough small $\zeta$ case. As a result, the existence regions of these motions mutually interweave to cause block segmentation which makes the regions of fundamental $1-p-p$ motions $(p \geq 2)$ present irregular distribution characteristics. Small $\zeta$ badly affects dynamical performance of the system, slightly large $\zeta$ leads to more energy consumption of the system to reduce its use efficiency. Based on comprehensive consideration of large existence region and low impact velocity of 1-1-1 motion, the damping ratio range $\zeta \in[0.1,0.2]$ is feasible and effective to the operation reliability of the system. Variation of the exciting force $f_{20}$ has an effect on the impact velocities and existence regions of $1-p-p$ motions and complete chattering-impact ones, but it hardly changes general forms of a series of periodic-impact motions. No anomaly type of periodic-impact motions occurs even for the extreme case of $f_{20}=0$ or 1 . As the restitution coefficient $R$ of impact is small enough, the existence region of complete chattering-impact motions is very large so that the regions of $1-1-1$ and $1-p-p(p \geq 2)$ motions are badly extruded and substantially reduced. The restitution coefficient of impact brings also great influences on the number of impacts and the sticking time of chattering motion in a motion period. As for large $R$, the impact sequence of chattering motion becomes more obvious in the motion period and the sticking time is correspondingly shorter due to slow attenuation in impacts. Conversely, as $R$ is relatively small, the impact strength is markedly attenuated so that the impact sequence of chattering motion decays quickly in the motion period and the sticking time becomes correspondingly longer.

\section{Acknowledgments}

The authors gratefully acknowledge the support by National Natural Science Foundation of China (11172119, 11362008), Innovative Research Group Foundation of Gansu Province of China (1308RJIA006) and Research Fund for the Doctoral Program of Higher Education of China (20126204110001). 


\section{References}

[1] S.W. Shaw, P.J. Holmes, A periodically forced piecewise linear oscillator, J. Sound Vib. 90 (1) (1983) 129-155.

[2] J.O. Aidanpää, B.R. Gupta, Periodic and chaotic behavior of a threshold-limited two-degree-of-freedom system, J. Sound Vib. 165 (2) (1993) 305-327.

[3] L.T. Silvio, de Souza, Iberê L. Caldas, Calculation of Lyapunov exponents in systems with impacts, Chaos, Solitons Fractals 19 (3) (2004) 569-579.

[4] F. Peterka, T. Kotera, S. Čipera, Explanation of appearance and characteristics of intermittency chaos of the impact oscillator, Chaos Solitons Fractals 19 (5) (2004) 1251-1259.

[5] Ekaterina Pavlovskaia, Marian Wiercigroch, Celso Grebogi, Modeling of an impact system with a drift, Phys. Rev. E 64 (5) (2001) 056224

[6] Ekaterina Pavlovskaia, Marian Wiercigroch, Celso Grebogi, Two-dimensional map for impact oscillator with drift, Phys. Rev. E 70 (3) (2004) 036201.

[7] D.D. Quinn, K. Bairavarasu, Near-simultaneous impacts, Int. J. Impact Eng. 32 (6) (2006) 889-904.

[8] Albert C.J. Luo, Period-doubling induced chaotic motion in the LR model of a horizontal impact oscillator, Chaos Solitons Fractals 19 (4) (2004) 823-839.

[9] R.I. Leine, Non-smooth stability analysis of the parametrically excited impact oscillator, Int. J. Non-Linear Mech. 47 (9) (2012) 1020-1032.

[10] Raouf A. Ibrahim, Vibro-impact dynamics: modeling, mapping and applications2009 ed.,Lecture Notes in Applied and Computational Mechanics, vol. 43, Springer, Berlin Heidelberg, 2009.

[11] A.B. Nordmark, Non-periodic motion caused by grazing incidence in an impact oscillator, J. Sound Vib. 145 (2) (1991) 279-297.

[12] G.S. Whiston, Singularities in vibro-impact dynamics, J. Sound Vib. 152 (3) (1992) 427-460.

[13] F. Peterka, J. Vacik, Transition to chaotic motion in mechanical systems with impacts, J. Sound Vib. 154 (1) (1992) 95-115.

[14] S. Foale, S.R. Bishop, Dynamical complexities of forced impacting systems, Philos. Trans. R. Soc. Lond. 338 (A) (1992) 547-556.

[15] A.P. Ivanov, Stabilization of an impact oscillator near grazing incidence owing to resonance, J. Sound Vib. 162 (3) (1993) 562-565.

[16] H.Y. Hu, Detection of grazing orbits and incident bifurcations of a forced continuous, piecewise-linear oscillator, J. Sound Vib. 187 (3) (1994) 485-493.

[17] J. de Weger, W. van de Water, J. Molenaar, Grazing impact oscillations, Phys. Rev. E 62 (2B) (2000) 2030-2041.

[18] M. di Bernardo, C.J. Budd, A.R. Champneys, Grazing and border-collision in piecewise-smooth systems: a unified analytical framework, Phys. Rev. Lett. 86 (12) (2001) 2553-2556.

[19] Albert C.J. Luo, Lidi Chen, Periodic motions and grazing in a harmonically forced, piecewise, linear oscillator with impacts, Chaos Solitons Fractals 24 (2) (2005) 567-578.

[20] Y. Ma, J. Ing, S. Banerjee, M. Wiercigroch, E. Pavlovskaia, The nature of the normal form map for soft impacting systems, Int. J. Non-Linear Mech. 43 (6) (2008) 504-513.

[21] S.L.T. de Souza, M. Wiercigroch, I.L. Caldas, J.M. Balthazar, Suppressing grazing chaos in impacting system by structural nonlinearity, Chaos Solitons Fractals 38 (3) (2008) 864-869.

[22] D. Chillingworth, Dynamics of an impact oscillator near a degenerate graze, Nonlinearity 23 (11) (2010) 2723-2748.

[23] N. Humphries, P.T. Piiroinen, A discontinuity-geometry view of the relationship between saddle-node and grazing bifurcations, Physica D 241 (22) (2012) 1911-1918.

[24] Harry Dankowicz, Michael Katzenbach, Discontinuity-induced bifurcations in models of mechanical contact, capillary adhesion, and cell division: a common framework, Physica D 241 (22) (2012) 1869-1881.

[25] Sergey Kryzhevich, Marian Wiercigroch, Topology of vibro-impact systems in the neighborhood of grazing, Physica D 241 (22) (2012) 1919-1931.

[26] C.J. Budd, F. Dux, Chattering and related behavior in impact oscillators, Philos. Trans. R. Soc. Lond. A 347 (1994) 365-389.

[27] C. Toulemonde, C. Gontier, Sticking motions of impact oscillators, Eur. J. Mech. A/Solids 17 (2) (1998) 339-366.

[28] Satoshi Ema, Etsuo Marui, Suppression of chatter vibration of boring tools using impact dampers, Int. J. Mach. Tools Manuf. 40 (8) (2000) 1141-1156.

[29] D.J. Wagg, Multiple non-smooth events in multi-degree-of-freedom vibroimpact systems, Nonlinear Dyn. 43 (2006) 137-148.

[30] D.J. Wagg, Rising phenomena and the multi-sliding bifurcation in a two-degree of freedom impact oscillator, Chaos Solitions Fractals 22 (3) (2003) 541-548.

[31] A.B. Nordmark, P.T. Piiroinen, Simulation and stability analysis of impacting systems with complete chattering, Nonlinear Dyn. 58 (2009) 85-106.

[32] L.T. Silvio, de Souza, Iberê L. Caldas, Basins of attraction and transient chaos in a gear-rattling model, J. Vib. Control 7 (6) (2001) 849-862.

[33] Albert C.J. Luo, Dennis O'Connor, Periodic motions and chaos with impacting chatter and stick in a gear transmission system, Int. J. Bifurc. Chaos 19 (6) (2009) 1975-1994.

[34] Albert C.J. Luo, Dennis O'Connor, Mechanism of impacting chatter with stick in a gear transmission system, Int. J. Bifurc. Chaos 19 (6) (2009) 2093-2105.

[35] Csaba Hős, Alan R. Champneys, Grazing bifurcations and chatter in a pressure relief valve model, Physica D 241 (2012) 2068-2076.

[36] G.L. Wen, Codimension-2 Hopf bifurcation of a two-degree-of-freedom vibroimpact system, J. Sound Vib. 242 (3) (2001) 475-485.

[37] W.C. Ding, J.H. Xie, Q.G. Sun, Interaction of Hopf and period doubling bifurcations of a vibro-impact system, J. Sound Vib. 275 (1-2) (2004) 27-45.
[38] G.W. Luo, Y.L. Zhang, J.G. Zhang, Codimension two bifurcations of fixed points in a class of vibratory systems with rigid stops, Nonlinear Anal.: Real World Appl. 8 (4) (2007) 1272-1292.

[39] J.F. Mason, N. Humphries, P.T. Piiroinen, Numerical analysis of codimensionone, -two and -three bifurcations in a periodically-forced impact oscillator with two discontinuity surfaces, Math. Comput. Simul. 95 (2014) 98-110.

[40] Harry Dankowicz, Xiaopeng Zhao, Local analysis of co-dimension-one and codimension-two grazing bifurcations in impact microactuators, Physica D 202 (3-4) (2005) 238-257.

[41] A.B. Nordmark, P. Kowalczyk, A codimension-two scenario of sliding solutions in grazing-sliding bifurcations, Nonlinearity 19 (2006) 1-26.

[42] M. Wiercigroch, V.T.W. Sin, Experimental study of a symmetrical piecewise base-excited oscillator, ASME J. Appl. Mech. 65 (3) (1998) 657-663.

[43] Dongping Jin, Haiyan Hu, An experimental study on possible types of vibroimpacts between two elastic beam, J. Exp. Mech. 14 (2) (1999) 129-135.

[44] D.D. Quinn, Finite duration impacts with external forces, J. Appl. Mech.: Trans, ASME 72 (5) (2005) 778-784.

[45] Nguyen Van-Du, Ko-Choong Woo, Ekaterina Pavlovskaia, Experimental study and mathematical modelling of a new of vibro-impact moling device, Int. J. Non-Linear Mech. 43 (6) (2008) 542-550.

[46] Elena Sitnikova, Ekaterina Pavlovskaia, James Ing, Experimental bifurcations of an impact oscillator with sma constraint, Int. J. Bifurc. Chaos 22 (5) (2012) 1230017.

[47] J.P. Meijaard, A.D. de Pater, Railway vehicle systems dynamics and chaotic vibrations, Int. J. Non-Linear Mech. 24 (1) (1989) 1-17.

[48] H. True, On the theory of nonlinear dynamics and its applications in vehicle systems dynamics, Veh. Syst. Dyn. 31 (5-6) (1999) 393-421.

[49] J.H. Xie, The mathematical model for the impact hammer and global bifurcations, Acta Mech. Sin. 29 (4) (1997) 456-463.

[50] Guanwei Luo, Xiaohong Lv, Dynamics of a plastic impact system with oscillatory and progressive motions, Int. J. Non-linear Mech. 43 (2) (2008) 100-110.

[51] Út V. Lê, A general mathematical model for the collision between a free-fall hammer of a pile-driver and an elastic pile: continuous dependence and lowfrequency asymptotic expansion, Nonlinear Anal.: Real World Appl. 12 (1) (2011) 702-722.

[52] Ekaterina Pavlovskaia, Marian Wiercigroch, Ko-Choong Woo, Albert A. Rodger, Modeling of ground moling dynamics by an impact oscillator with a frictional slider, Meccanica 38 (1) (2003) 85-97.

[53] Haoran Liu, Fei Liu, Dongxiao Hou, Vibration characteristics of mill rolls under multi-segment nonlinear elastic constraints, Chin. J. Mech. Eng. 48 (9) (2012) 89-94.

[54] A. Bibó, Gy. Kórolyi, T. Bódai, Fly-wheel model exhibits the hither and thither motion of a bouncing ball, Int. J. Non-Linear Mech. 44 (8) (2009) 905-912.

[55] Z. Li, L. Li, S.X. Bai, A new method of predicting the occurrence of contact loss between pairing linkages with clearances, Mech. Mach. Theory 27 (3) (1992) 295-301.

[56] M. Wiercigroch, R.D. Neilson, M.A. Player, Material removal rate prediction for ultrasonic drilling of hard materials using an impact oscillator approach, Phys. Lett. A 259 (2) (1999) 91-96.

[57] M. Wiercigroch, J. Wojewoda, A.M. Krivtsov, Dynamics of ultrasonic percussive drilling of hard rocks, J. Sound Vib. 280 (3-5) (2005) 739-757.

[58] Yang Liu, Ekaterina Pavlovskaia, David Hendry, Marian Wiercigroch, Vibroimpact responses of capsule system with various friction models, Int. J. Mech. Sci. 72 (2013) 39-54

[59] Wei Li, Yi Yang, Deren Sheng, A novel nonlinear model of rotor/bearing/seal system and numerical analysis, Mech. Mach. Theory 46 (5) (2011) 618-631.

[60] X.L. An, J.Z. Zhou, X.Q. Xiang, et al., Dynamic response of a rub-impact rotor system under axial thrust, Arch. Appl. Mech. 79 (11) (2009) 1009-1018.

[61] E.E. Pavlovskaia, E.V. Karpenko, M. Wiercigroch, Non-linear dynamic interactions of a Jeffcott rotor with preloaded snubber ring, J. Sound Vib. 276 (1-2) (2004) 361-379.

[62] Aref Afsharfard, Anooshiravan Farshidianfar, Design of nonlinear impact dampers based on acoustic and damping behavior, Int. J. Mech. Sci. 65 (1) (2012) 125-133.

[63] Yanchen Du, Shulin Wang, Modeling the fine particle impact damper, Int. J. Mech. Sci. 52 (7) (2010) 1015-1022.

[64] D.D. Quinn, The dynamics of two parametrically excited pendula with impacts, Int. J. Bifurc. Chaos 15 (6) (2005) 1975-1988.

[65] Ekaterina Pavlovskaia, David C. Hendry, Marian Wiercigroch. Modelling of high frequency vibro-impact drilling. Int. J. Mech. Sci. (2013), http://dx.doi.org/ 10.1016/j.ijmecsci.2013.08.009.

[66] Raouf A. Ibrahim, Overview of vibro-impact dynamics of pipes conveying fluids and the problem of fluid-elastic instability, in: Proceedings of the ASME Pressure Vessels and Piping Conference, Prague, Czech Republic, C. Giannopapa (Ed.), vol. 4, 2009, pp. 127-137.

[67] Ihab M. Grace, Raouf A. Ibrahim, Valery N. Pilipchuk, Inelastic impact dynamics of ships with one-sided barriers. Part I: analytical and numerical investigations, Nonlinear Dyn. 66 (4) (2011) 589-607.

[68] Albert C.J. Luo, Dennis O'Connor, Periodic motions with impacting chatter and stick in a gear transmission system, J. Vib. Acoust.: Trans. ASME 131 (4) (2009) 041013.

[69] Byrtus Miroslav, Zeman Vladimir, On modeling and vibration of gear drives influenced by nonlinear couplings, Mech. Mach. Theory 46 (3) (2011) 375-397.

[70] M. Ognjanovic, F. Agemi, Gear vibrations in supercritical mesh-frequency range caused by teeth impacts, J. Mech. Eng. 56 (10) (2010) 653-662.

[71] Joanna F. Mason, Petri T. Piiroinen, R. Eddie Wilson, Basins of attraction in nonsmooth models of gear rattle, Int. J. Bifurc. Chaos 19 (1) (2009) 203-224. 